\title{
Artin monoids inject in their groups
}

Luis Paris

\begin{abstract}
We prove that the natural homomorphism from an Artin monoid to its associated Artin group is always injective.
\end{abstract}

Mathematics Subject Classification (2000). Primary 20F36; Secondary 20F55.

Keywords. Artin groups, Artin monoids, linear representations.

\section{Introduction}

Let $S$ be a finite set. A Coxeter matrix over $S$ is a matrix $M=\left(m_{s, t}\right)_{s, t \in S}$ indexed by the elements of $S$ and such that:

- $m_{s, s}=1$ for all $s \in S$;

- $m_{s, t}=m_{t, s} \in\{2,3,4, \ldots,+\infty\}$ for all $s, t \in S, s \neq t$.

A Coxeter matrix $M=\left(m_{s, t}\right)_{s, t \in S}$ is usually represented by its Coxeter graph $\Gamma$. This is defined by the following data:

- $\mathrm{S}$ is the set of vertices of $\Gamma$;

- two vertices $s, t \in S$ are joined by an edge if $m_{s, t} \geq 3$;

- the edge which joins $s$ and $t$ is labeled by $m_{s, t}$ if $m_{s, t} \geq 4$.

The Coxeter system associated with $\Gamma$ is the pair $(W, S)$, where $W$ is the group presented by

$$
\left.W=\langle S| s^{2}=1 \text { for } s \in S,(s t)^{m_{s, t}}=1 \text { for } s, t \in S, s \neq t, m_{s, t}<+\infty\right\rangle .
$$

The group $W$ is called the Coxeter group associated with $\Gamma$.

Let $\Sigma=\left\{\sigma_{s} ; s \in S\right\}$ be an abstract set in one-to-one correspondence with $S$. For two objects $a, b$ and $m \in \mathbf{N}$ we write

$$
\operatorname{prod}(a, b ; m)= \begin{cases}(a b)^{\frac{m}{2}} & \text { if } m \text { is even } \\ (a b)^{\frac{m-1}{2}} a & \text { if } m \text { is odd }\end{cases}
$$


The Artin system associated with $\Gamma$ is the pair $\left(G_{\Gamma}, \Sigma\right)$, where $G_{\Gamma}$ is the group presented by

$$
\left.G_{\Gamma}=\langle\Sigma| \operatorname{prod}\left(\sigma_{s}, \sigma_{t} ; m_{s, t}\right)=\operatorname{prod}\left(\sigma_{t}, \sigma_{s} ; m_{s, t}\right) \text { for } s, t \in S, s \neq t, m_{s, t}<+\infty\right\rangle .
$$

The group $G_{\Gamma}$ is called the Artin group associated with $\Gamma$.

Recall that a monoid is a semigroup with a unity, and a homomorphism of monoids is a map $\phi: M \rightarrow M^{\prime}$ which satisfies $\phi(f g)=\phi(f) \phi(g)$ for all $f, g \in M$, and $\phi(1)=1$. The Artin monoid associated with $\Gamma$ is the monoid $G_{\Gamma}^{+}$presented by the same generators and relations as $G_{\Gamma}$. Let $\iota: G_{\Gamma}^{+} \rightarrow G_{\Gamma}$ denote the canonical homomorphism from $G_{\Gamma}^{+}$to $G_{\Gamma}$. The goal of this paper is to prove the following.

Theorem 1.1. The homomorphism $\iota: G_{\Gamma}^{+} \rightarrow G_{\Gamma}$ is injective for all Coxeter graphs.

It seems that the authorship of the Artin groups, also called generalized braid groups, has to be attributed to Jacques Tits, in spite of the fact that his name does not always appear in the references. Furthermore, it is in a paper of him [Tit2] where these groups appeared for the first time. However, it was Brieskorn and Saito who proposed in [BS] the question of the study of all these groups (Deligne's paper [Del], which appeared at the same time, is concerned only with spherical type Artin groups, namely, those Artin groups for which $W$ is finite). Some families of Artin groups are well understood, but, since the paper of Brieskorn and Saito in 1972, very few results concerning all Artin groups have been published. In particular, Theorem 1.1 above was known only for some particular classes, namely, for the spherical type Artin groups (see [BS] and [Del]), for the two-dimensional Artin groups (see $[\mathrm{ChP}]$ and [Cha]), and for the FC-type Artin groups (see [Alt] and [Cha]). It was unknown, for example, for the so-called affine type Artin groups.

Our proof of Theorem 1.1 is independent of the previous approaches of the problem. Note first that, in order to prove Theorem 1.1, it suffices to show that there exists an injective homomorphism $\psi: G_{\Gamma}^{+} \rightarrow G$, where $G$ is a group, not necessarily equal to $G_{\Gamma}$.

We say that a Coxeter graph $\Gamma$ is of small type if $m_{s, t} \in\{2,3\}$ for all $s, t \in S$, $s \neq t$. We say that $\Gamma$ has no triangle if there is no triple $\{s, t, r\}$ in $S$ such that $m_{s, t}, m_{s, r}, m_{t, r} \geq 3$. The first ingredient in our proof is to show that, for any Coxeter graph $\Gamma$, there exists an injective homomorphism $\phi: G_{\Gamma}^{+} \rightarrow G_{\tilde{\Gamma}}^{+}$, where $G_{\tilde{\Gamma}}^{+}$is an Artin monoid associated to a Coxeter graph $\tilde{\Gamma}$ of small type with no triangle. The homomorphism $\phi$ is obtained by a "folding" as described in [Cri], its construction is essentially the same as the one given in $[\mathrm{CrP}$, Sec. 6], and the proof of the injectivity is a direct application of [Cri, Thm. 1.3]. This construction is given in Section 5.

So, in order to prove Theorem 1.1, it suffices to consider only Coxeter graphs of small type with no triangle. Take such a Coxeter graph, $\Gamma$. We construct in 
Section 3 a homomorphism $\psi: G_{\Gamma}^{+} \rightarrow \operatorname{Gl}(V)$, where $V$ is a (infinite dimensional) vector space over $\mathbf{Q}(x, y)$, and we prove in Section 4 that $\psi$ is injective.

If $\Gamma=A_{n}$, then $G_{A_{n}}$ is the braid group on $n+1$ strings, and $\psi: G_{A_{n}}^{+} \rightarrow$ $\mathrm{Gl}(V)$ is equivalent to the representation constructed by Bigelow and Krammer in [Big], [Kra1] and [Kra2]. In this case, $V$ has finite dimension, and the injectivity of $\psi$ implies the injectivity of the induced representation $G_{A_{n}} \rightarrow \operatorname{Gl}(V)$. More generally, if $\Gamma$ is of spherical and small type, then the representation $\psi: G_{\Gamma}^{+} \rightarrow$ $\mathrm{Gl}(V)$ is equivalent to the ones constructed independently by Digne [Dig], and by Cohen and Wales [CW]. In this case again, $V$ has finite dimension and the induced representation $G_{\Gamma} \rightarrow \mathrm{Gl}(V)$ is injective. We do not know whether the representation $G_{\Gamma} \rightarrow \operatorname{Gl}(V)$ induced by $\psi$ is injective for all Coxeter graphs of small type with no triangle. The construction of $\psi$ and the proof of the injectivity are based on a (non always easy) generalization of the methods of Krammer, Digne, Cohen and Wales.

Acknowledgments. I would like to thank John Crisp for many useful conversations during the preparation of this work, and for drawing my attention to the results of [Cri] which are one of the main tools of the proof of Theorem 1.1.

\section{Preliminaries}

We summarize in this section some well known results on Artin monoids, Coxeter groups and root systems, and give definitions and some basic properties of closed sets. The closed sets have been introduced by Krammer in [Kra2] for Artin groups of type $A_{n}$. This notion has been extended to the Artin groups of small and spherical type by Digne [Dig], Cohen and Wales $[\mathrm{CW}]$. Here we extend it to all small type Artin groups.

Let $\Gamma$ be a Coxeter graph. It is shown in [BS] that the Artin monoid $G_{\Gamma}^{+}$is cancellative, namely, if $f g_{1} h=f g_{2} h$, then $g_{1}=g_{2}$. We say that $h$ is a multiple of $g$ and write $g<h$ if there exists $f \in G_{\Gamma}^{+}$such that $g f=h$. The relation $<$ is a partial ordering on $G_{\Gamma}^{+}$.

Let $\theta: G_{\Gamma}^{+} \rightarrow W$ be the homomorphism which sends $\sigma_{s}$ to $s$ for all $s \in S$. Then $\theta$ has a natural set-section $\tau: W \rightarrow G_{\Gamma}^{+}$defined as follows. Let $w \in W$. We choose a reduced expression $w=s_{1} \ldots s_{l}$ for $w$ and we set $\tau(w)=\sigma_{s_{1}} \ldots \sigma_{s_{l}}$. By Tits' solution of the word problem for Coxeter groups [Tit1], the definition of $\tau(w)$ does not depend on the choice of the reduced expression.

Let $l: W \rightarrow \mathbf{N}$ and $l: G_{\Gamma}^{+} \rightarrow \mathbf{N}$ denote the word length functions of $W$ and $G_{\Gamma}^{+}$with respect to $S$ and $\Sigma$, respectively. Define a partial ordering on $W$ by $u<v$ if $l(v)=l(u)+l\left(u^{-1} v\right)$. Then $l(\tau(w))=l(w)$ for all $w \in W$, and one has $u<v$ if and only if $\tau(u)<\tau(v)$.

The proof of the following proposition is essentially the same as the one of [Del, Pro. 1.14] and [Mic, Lem. 1.4]. 
Proposition 2.1. Let $E$ be a nonempty finite subset of $W$ such that:

- if $u<v$ and $v \in E$, then $u \in E$;

- if $v \in W$ and $s, t \in S$ are such that $l(v s)=l(v t)=l(v)+1$ and $v s, v t \in E$, then $m_{s, t}<+\infty$ and $v \cdot \operatorname{prod}\left(s, t ; m_{s, t}\right) \in E$.

Then there exists $w_{0} \in W$ such that $E=\left\{v \in W ; v<w_{0}\right\}$.

The next proposition is part of [Mic, Prop. 2.1]. It is also a direct consequence of [BS, Lem. 2.1 and Prop. 2.3].

Proposition 2.2. Let $f \in G_{\Gamma}^{+}$and let $E=\{w \in W ; \tau(w)<f\}$. Then $E$ satisfies:

- if $u<v$ and $v \in E$, then $u \in E$;

- if $v \in W$ and $s, t \in S$ are such that $l(v s)=l(v t)=l(v)+1$ and $v s, v t \in E$, then $m_{s, t}<+\infty$ and $v \cdot \operatorname{prod}\left(s, t ; m_{s, t}\right) \in E$.

Definition. Let $f \in G_{\Gamma}^{+}$. By Propositions 2.1 and 2.2, there exists a unique $w_{0} \in W$ such that $\{v \in W ; \tau(v)<f\}=\left\{v \in W ; v<w_{0}\right\}$. We set

$$
L(f)=w_{0} .
$$

The next proposition is also part of [Mic, Prop. 2.1]

Proposition 2.3. Let $f, g \in G_{\Gamma}^{+}$. Then

$$
L(f g)=L(f \cdot(\tau \circ L)(g)) .
$$

Let $\Pi=\left\{\alpha_{s} ; s \in S\right\}$ be an abstract set in one-to-one correspondence with $S$. The elements of $\Pi$ are called simple roots. Let $U$ denote the real vector space having $\Pi$ as a basis, and let $\langle\rangle:, U \times U \rightarrow \mathbf{R}$ be the symmetric bilinear form on $U$ defined by

$$
\left\langle\alpha_{s}, \alpha_{t}\right\rangle= \begin{cases}-2 \cos \left(\pi / m_{s, t}\right) & \text { if } m_{s, t}<+\infty \\ -2 & \text { if } m_{s, t}=+\infty\end{cases}
$$

There is a faithful representation $W \rightarrow \mathrm{Gl}(U)$ which is defined by

$$
s(x)=x-\left\langle\alpha_{s}, x\right\rangle \alpha_{s}, \quad x \in U, s \in S,
$$

and which preserves the bilinear form $\langle$,$\rangle . This representation is called the$ canonical representation of $W$.

The set $\Phi=\left\{w \alpha_{s} ; s \in S, w \in W\right\}$ is called the root system of $W$. The subsets $\Phi^{+}=\left\{\sum_{s \in S} \lambda_{s} \alpha_{s} \in \Phi ; \lambda_{s} \geq 0\right.$ for all $\left.s \in S\right\}$ and $\Phi^{-}=\left\{\beta \in \Phi ;-\beta \in \Phi^{+}\right\}$ are the sets of positive roots and negative roots, respectively. For $w \in W$ we set $\Phi_{w}=\left\{\beta \in \Phi^{+} ; w^{-1} \beta \in \Phi^{-}\right\}$. 
We list in the following proposition some well known results on root systems (see [Hil] and [Deo]).

Proposition 2.4. (1) $\Phi=\Phi^{+} \sqcup \Phi^{-}$.

(2) $\left|\Phi_{w}\right|=l(w)$ for all $w \in W$.

(3) For all $u, v \in W$ such that $u<v$, one has $\Phi_{v}=\Phi_{u} \sqcup u \cdot \Phi_{u^{-1} v}$.

(4) For all $w \in W$ and $s \in S$,

$$
l(s w)= \begin{cases}l(w)+1 & \text { if } w^{-1} \alpha_{s} \in \Phi^{+}, \\ l(w)-1 & \text { if } w^{-1} \alpha_{s} \in \Phi^{-} .\end{cases}
$$

(5) Let $\beta=w \alpha_{s} \in \Phi^{+}$, and let $r_{\beta}=w s w^{-1}$. Then $r_{\beta}$ acts on $U$ by

$$
r_{\beta}(x)=x-\langle x, \beta\rangle \beta, \quad x \in U .
$$

Let $\beta \in \Phi^{+}$. Define the depth of $\beta$ to be

$\operatorname{dp}(\beta)=\min \left\{l \in \mathbf{N}\right.$; there exists $w \in W$ such that $w \beta \in \Phi^{-}$and $\left.l(w)=l\right\}$.

Lemma 2.5. Let $\beta \in \Phi^{+}$. Then

$$
\begin{aligned}
& \operatorname{dp}(\beta)=\min \{l \in \mathbf{N} ; \text { there exist } w \in W \text { and } s \in S \\
& \left.\qquad \text { such that } \beta=w^{-1} \alpha_{s} \text { and } l=l(w)+1\right\} .
\end{aligned}
$$

Proof. Let $d_{1}=\min \left\{l \in \mathbf{N}\right.$; there exists $w \in W$ such that $w \beta \in \Phi^{-}$and $\left.l(w)=l\right\}$ and $d_{2}=\min \left\{l \in \mathbf{N}\right.$; there exist $w \in W$ and $s \in S$ such that $\beta=w^{-1} \alpha_{s}$ and $l=l(w)+1\}$.

Let $w \in W$ and $s \in S$ such that $\beta=w^{-1} \alpha_{s}$ and $l(w)=d_{2}-1$. Since $\beta \in \Phi^{+}$, by Proposition 2.4, $l(s w)=l(w)+1=d_{2}$. Moreover, $s w \beta=s \alpha_{s}=-\alpha_{s} \in \Phi^{-}$. This shows that $d_{2} \leq d_{1}$.

Let $w \in W$ such that $w \beta \in \Phi^{-}$and $l(w)=d_{1}$. Let $s \in S$ such that $l(s w)=$ $l(w)-1$. Let $v=s w$ and $\gamma=v \beta$. By the minimality of $l(w)=d_{1}$, one has $\gamma \in \Phi^{+}$. Moreover, $s \gamma=w \beta \in \Phi^{-}$, thus $\gamma=\alpha_{s}$ and $\beta=v^{-1} \alpha_{s}$. This shows that $d_{1} \leq d_{2}$.

The following proposition is proved in [BH, Lem. 1.7].

Proposition 2.6. Let $s \in S$ and $\beta \in \Phi^{+} \backslash\left\{\alpha_{s}\right\}$. Then

$$
\operatorname{dp}(s \cdot \beta)= \begin{cases}\operatorname{dp}(\beta)-1 & \text { if }\left\langle\alpha_{s}, \beta\right\rangle>0 \\ \operatorname{dp}(\beta) & \text { if }\left\langle\alpha_{s}, \beta\right\rangle=0, \\ \operatorname{dp}(\beta)+1 & \text { if }\left\langle\alpha_{s}, \beta\right\rangle<0 .\end{cases}
$$


From now on and till the end of the section, we assume that $\Gamma$ is a Coxeter graph of small type, namely, that $m_{s, t} \in\{2,3\}$ for all $s, t \in S, s \neq t$. Note that, under this assumption, all the roots can be written $\beta=\sum_{s \in S} \lambda_{s} \alpha_{s}$, with $\lambda_{s} \in \mathbf{Z}$, and one has $\langle\beta, \gamma\rangle \in \mathbf{Z}$ for all $\beta, \gamma \in \Phi$.

Definition. A subset $A \subset \Phi^{+}$is a closed subset if:

- $A$ is finite;

- if $\alpha, \beta \in A$, then $\langle\alpha, \beta\rangle \geq-1$;

- if $\alpha, \beta \in A$ and $\langle\alpha, \beta\rangle=-1$, then $\alpha+\beta=r_{\alpha}(\beta)=r_{\beta}(\alpha) \in A$.

Lemma 2.7. Let $w \in W$. Then $\Phi_{w}$ is a closed subset.

Proof. Let $\alpha, \beta \in \Phi^{+}$. A direct calculation shows that: if $\langle\alpha, \beta\rangle \leq-2$, then $\left(r_{\alpha} r_{\beta}\right)^{l}(\alpha)$ is a positive root of the form $a_{l} \alpha+b_{l} \beta$, where $a_{l}, b_{l} \geq 0$, for all $l \in \mathbf{N}$, and $\left(r_{\alpha} r_{\beta}\right)^{l}(\alpha) \neq\left(r_{\alpha} r_{\beta}\right)^{k}(\alpha)$ for $l \neq k$. This implies that: if $\langle\alpha, \beta\rangle \leq-2$, then there are infinitely many positive roots of the form $a \alpha+b \beta$, with $a, b \geq 0$.

The set $\Phi_{w}$ is finite since $\left|\Phi_{w}\right|=l(w)$. Let $\alpha, \beta \in \Phi_{w}$. If $\gamma=a \alpha+b \beta$, with $a, b \geq 0$, is a positive root, then $\gamma \in \Phi_{w}$, since $w^{-1} \gamma=a w^{-1} \alpha+b w^{-1} \beta$ is a negative root. By the above considerations, this implies that $\langle\alpha, \beta\rangle \geq-1$ and that $\alpha+\beta \in \Phi_{w}$ if $\langle\alpha, \beta\rangle=-1$.

Proposition 2.8. Let $A$ be a closed subset of $\Phi^{+}$and let $E=\left\{w \in W ; \Phi_{w} \subset A\right\}$. Then E satisfies:

- $E$ is finite;

- if $u<v$ and $v \in E$, then $u \in E$;

- if $v \in W$ and $s, t \in S$ are such that $l(v s)=l(v t)=l(v)+1$ and $v s, v t \in E$, then $v \cdot \operatorname{prod}\left(s, t ; m_{s, t}\right) \in E$.

Proof. If $\Phi_{w} \subset A$, then $l(w)=\left|\Phi_{w}\right| \leq|A|$. Since $A$ is finite, it follows that $l(w)$ is bounded for all $w \in E$, thus $E$ is finite.

Suppose $u<v$ and $v \in E$. Then, by Proposition 2.4, $\Phi_{u} \subset \Phi_{v} \subset A$, thus $u \in E$.

Let $v \in W$ and $s, t \in S$ such that $l(v s)=l(v t)=l(v)+1$ and $v s, v t \in E$. By Proposition 2.4, one has $\Phi_{v s}=\Phi_{v} \cup\left\{v \alpha_{s}\right\}$ and $\Phi_{v t}=\Phi_{v} \cup\left\{v \alpha_{t}\right\}$. Let $w=v \cdot \operatorname{prod}\left(s, t ; m_{s, t}\right)$. If $m_{s, t}=2$ then $\Phi_{w}=\Phi_{v} \cup\left\{v \alpha_{s}, v \alpha_{t}\right\} \subset A$, thus $w \in E$. If $m_{s, t}=3$, then $\left\langle v \alpha_{s}, v \alpha_{t}\right\rangle=\left\langle\alpha_{s}, \alpha_{t}\right\rangle=-1$, thus $v \alpha_{s}+v \alpha_{t}=v\left(\alpha_{s}+\alpha_{t}\right) \in A$. It follows that $\Phi_{w}=\Phi_{v} \cup\left\{v \alpha_{s}, v \alpha_{t}, v\left(\alpha_{s}+\alpha_{t}\right)\right\} \subset A$, thus $w \in E$.

Definition. Let $A$ be a closed subset of $\Phi^{+}$. By Propositions 2.1 and 2.8, there exists a unique $w_{0} \in W$ such that $\left\{w \in W ; \Phi_{w} \subset A\right\}=\left\{w \in W ; w<w_{0}\right\}$. We set

$$
C(A)=w_{0}
$$

Note that $C\left(\Phi_{w}\right)=w$ for all $w \in W$. 


\section{The representation}

Throughout this section, $\Gamma$ is assumed to be a Coxeter graph of small type with no triangle, namely, $m_{s, t} \in\{2,3\}$ for all $s, t \in S, s \neq t$, and there is no triple $\{s, t, r\}$ in $S$ such that $m_{s, t}=m_{s, r}=m_{t, r}=3$. Our aim here is to construct a (infinite dimensional) linear representation $\psi: G_{\Gamma}^{+} \rightarrow \mathrm{Gl}(V)$. We will prove in Section 4 that this linear representation is faithful. This will imply that $\iota: G_{\Gamma}^{+} \rightarrow G_{\Gamma}$ is injective.

Let $\mathcal{E}=\left\{e_{\beta} ; \beta \in \Phi^{+}\right\}$be an abstract set in one-to-one correspondence with $\Phi^{+}$, let $\mathbf{K}=\mathbf{Q}(x, y)$ denote the field of rational functions on two variables over $\mathbf{Q}$, and let $V$ be the $\mathbf{K}$-vector space having $\mathcal{E}$ as a basis.

For all $s \in S$, we define a linear transformation $\varphi_{s}: V \rightarrow V$ by

$$
\varphi_{s}\left(e_{\beta}\right)= \begin{cases}0 & \text { if } \beta=\alpha_{s} \\ e_{\beta} & \text { if }\left\langle\alpha_{s}, \beta\right\rangle=0 \\ y \cdot e_{\beta-a \alpha_{s}} & \text { if }\left\langle\alpha_{s}, \beta\right\rangle=a>0 \text { and } \beta \neq \alpha_{s} \\ (1-y) \cdot e_{\beta}+e_{\beta+a \alpha_{s}} & \text { if }\left\langle\alpha_{s}, \beta\right\rangle=-a<0\end{cases}
$$

A direct (case by case) calculation shows that

$$
\begin{aligned}
\varphi_{s} \varphi_{t} & =\varphi_{t} \varphi_{s} & & \text { if } m_{s, t}=2, \\
\varphi_{s} \varphi_{t} \varphi_{s} & =\varphi_{t} \varphi_{s} \varphi_{t} & & \text { if } m_{s, t}=3 .
\end{aligned}
$$

So:

Proposition 3.1. The mapping $\sigma_{s} \rightarrow \varphi_{s}, s \in S$, induces a homomorphism $\varphi$ : $G_{\Gamma}^{+} \rightarrow \operatorname{End}(V)$.

Now, for all $s \in S$ and all $\beta \in \Phi^{+}$, take a polynomial $T(s, \beta) \in \mathbf{Q}[y]$ and define $\psi_{s}: V \rightarrow V$ by

$$
\psi_{s}\left(e_{\beta}\right)=\varphi_{s}\left(e_{\beta}\right)+x T(s, \beta) \cdot e_{\alpha_{s}} .
$$

The goal of this section is to prove the following:

Theorem 3.2. There is a choice of polynomials $T(s, \beta), s \in S$ and $\beta \in \Phi^{+}$, so that the mapping $\sigma_{s} \rightarrow \psi_{s}, s \in S$, induces a homomorphism $\psi: G_{\Gamma}^{+} \rightarrow \operatorname{Gl}(V)$.

Let $s \in S$ and $\beta \in \Phi^{+}$. We define the polynomial $T(s, \beta)$ by induction on $\operatorname{dp}(\beta)$. Assume first that $\operatorname{dp}(\beta)=1$. There exists $t \in S$ such that $\beta=\alpha_{t}$. Then we set

(D1) $T\left(s, \alpha_{t}\right)=y^{2} \quad$ if $t=s$,

(D2) $T\left(s, \alpha_{t}\right)=0 \quad$ if $t \neq s$. 
Now, assume that $\operatorname{dp}(\beta) \geq 2$. We choose $t \in S$ such that $\operatorname{dp}(t \cdot \beta)=\operatorname{dp}(\beta)-1$. By Proposition 2.6, one has $\left\langle\alpha_{t}, \beta\right\rangle=b>0$.

Case 1: $\left\langle\alpha_{s}, \beta\right\rangle=a>0$. Then we set

(D3) $T(s, \beta)=y^{\operatorname{dp}(\beta)}(y-1)$.

Case 2: $\left\langle\alpha_{s}, \beta\right\rangle=0$. Then we set

(D4) $T(s, \beta)=y \cdot T\left(s, \beta-b \alpha_{t}\right)$ if $\left\langle\alpha_{s}, \alpha_{t}\right\rangle=0$,

(D5) $T(s, \beta)=(y-1) \cdot T\left(s, \beta-b \alpha_{t}\right)+y \cdot T\left(t, \beta-b \alpha_{s}-b \alpha_{t}\right)$ if $\left\langle\alpha_{s}, \alpha_{t}\right\rangle=-1$

Case 3: $\left\langle\alpha_{s}, \beta\right\rangle=-a<0$. Then we set

(D6) $T(s, \beta)=y \cdot T\left(s, \beta-b \alpha_{t}\right)$ if $\left\langle\alpha_{s}, \alpha_{t}\right\rangle=0$

(D7) $T(s, \beta)=(y-1) \cdot T\left(s, \beta-b \alpha_{t}\right)$ $+y \cdot T\left(t, \beta-(b-a) \alpha_{s}-b \alpha_{t}\right) \quad$ if $\left\langle\alpha_{s}, \alpha_{t}\right\rangle=-1$ and $b>a$

(D8) $T(s, \beta)=T\left(t, \beta-b \alpha_{t}\right)$

$$
+(y-1) \cdot T\left(s, \beta-b \alpha_{t}\right) \quad \text { if }\left\langle\alpha_{s}, \alpha_{t}\right\rangle=-1 \text { and } b=a
$$

(D9) $T(s, \beta)=y \cdot T\left(s, \beta-b \alpha_{t}\right)$

$$
+T\left(t, \beta-b \alpha_{t}\right)+y^{\mathrm{dp}(\beta)-1}(1-y) \quad \text { if }\left\langle\alpha_{s}, \alpha_{t}\right\rangle=-1 \text { and } b<a .
$$

The proofs of the following lemmas 3.3 and 3.4 are long and tedious case by case verifications and they are not very instructive for the remainder of the paper. So, we put them in a separate section at the end of the paper and continue with the proof of Theorem 3.2.

Lemma 3.3. Let $s \in S$ and $\beta \in \Phi^{+}$such that $\operatorname{dp}(\beta) \geq 2$ and $\left\langle\alpha_{s}, \beta\right\rangle=0$. Then the definition of $T(s, \beta)$ does not depend on the choice of the $t \in S$ such that $\operatorname{dp}(t \cdot \beta)=\operatorname{dp}(\beta)-1$.

Lemma 3.4. Let $s \in S$ and $\beta \in \Phi^{+}$such that $\operatorname{dp}(\beta) \geq 2$ and $\left\langle\alpha_{s}, \beta\right\rangle=-a<0$. Then the definition of $T(s, \beta)$ does not depend on the choice of the $t \in S$ such that $\operatorname{dp}(t \cdot \beta)=\operatorname{dp}(\beta)-1$.

Lemma 3.5. Let $s, t \in S$ and $\beta \in \Phi^{+}$such that $\left\langle\alpha_{s}, \alpha_{t}\right\rangle=-1,\left\langle\alpha_{s}, \beta\right\rangle=0$, and $\left\langle\alpha_{t}, \beta\right\rangle=0$. Then

$$
T(s, \beta)=T(t, \beta) \text {. }
$$

Proof. We argue by induction on $\operatorname{dp}(\beta)$. Assume first that $\operatorname{dp}(\beta)=1$. There exists $r \in S$ such that $\beta=\alpha_{r}$. One has $r \neq s$ and $r \neq t$ since $\left\langle\alpha_{s}, \beta\right\rangle=\left\langle\alpha_{t}, \beta\right\rangle=0$. Then, by (D2),

$$
T(s, \beta)=T\left(s, \alpha_{r}\right)=0=T\left(t, \alpha_{r}\right)=T(t, \beta) .
$$


Now, assume that $\operatorname{dp}(\beta) \geq 2$. We choose $r \in S$ such that $\operatorname{dp}(r \cdot \beta)=\operatorname{dp}(\beta)-1$. By Proposition 2.6, one has $\left\langle\alpha_{r}, \beta\right\rangle=c>0$.

Case 1: $\left\langle\alpha_{s}, \alpha_{r}\right\rangle=0$ and $\left\langle\alpha_{t}, \alpha_{r}\right\rangle=0$. Then

$$
\begin{aligned}
T(s, \beta) & =y \cdot T\left(s, \beta-c \alpha_{r}\right) & & \text { by (D4) } \\
& =y \cdot T\left(t, \beta-c \alpha_{r}\right) & & \text { by induction } \\
& =T(t, \beta) & & \text { by (D4). }
\end{aligned}
$$

Case 2: $\left\langle\alpha_{s}, \alpha_{r}\right\rangle=0$ and $\left\langle\alpha_{t}, \alpha_{r}\right\rangle=-1$. We cannot have $\operatorname{dp}(\beta) \leq 3$ in this case. Suppose $\operatorname{dp}(\beta) \geq 4$. Then

$$
\begin{aligned}
T(s, \beta)= & y \cdot T\left(s, \beta-c \alpha_{r}\right) & & \text { by (D4) } \\
= & y(y-1) \cdot T\left(s, \beta-c \alpha_{t}-c \alpha_{r}\right) & & \\
& +y^{2} \cdot T\left(t, \beta-c \alpha_{s}-c \alpha_{t}-c \alpha_{r}\right) & & \text { by (D5) } \\
= & y^{\operatorname{dp}(\beta)-1}(y-1)^{2}+y^{2} \cdot T\left(t, \beta-c \alpha_{s}-c \alpha_{t}-c \alpha_{r}\right) & & \text { by (D3) } \\
= & y^{\operatorname{dp}(\beta)-1}(y-1)^{2}+y^{2} \cdot T\left(r, \beta-c \alpha_{s}-c \alpha_{t}-c \alpha_{r}\right) & & \text { by induction } \\
= & (y-1) \cdot T\left(t, \beta-c \alpha_{r}\right)+y \cdot T\left(r, \beta-c \alpha_{t}-c \alpha_{r}\right) & & \text { by (D3) and (D4) } \\
= & T(t, \beta) & & \text { by (D5). }
\end{aligned}
$$

Since $\Gamma$ has no triangle, we cannot have $\left\langle\alpha_{s}, \alpha_{r}\right\rangle=-1$ and $\left\langle\alpha_{t}, \alpha_{r}\right\rangle=-1$ (because $\left\langle\alpha_{s}, \alpha_{t}\right\rangle=-1$ ). So, Case 1 and Case 2 are the only possible cases.

Lemma 3.6. Let $s, t \in S$ such that $m_{s, t}=2$. Then $\psi_{s} \psi_{t}=\psi_{t} \psi_{s}$.

Proof. Let $\beta \in \Phi^{+}$. We compute $\left(\psi_{s} \psi_{t}\right)\left(e_{\beta}\right)$ and $\left(\psi_{t} \psi_{s}\right)\left(e_{\beta}\right)$ replacing $T\left(s, \alpha_{s}\right)$ and $T\left(t, \alpha_{t}\right)$ by $y^{2}$, and replacing $T\left(s, \alpha_{t}\right)$ and $T\left(t, \alpha_{s}\right)$ by 0 , and we compare both expressions. This can be easily made with a computer.

Case 1: $\beta=\alpha_{s}$. Then we directly obtain $\left(\psi_{s} \psi_{t}\right)\left(e_{\beta}\right)=\left(\psi_{t} \psi_{s}\right)\left(e_{\beta}\right)$.

Case 2: $\left\langle\alpha_{s}, \beta\right\rangle=0$ and $\left\langle\alpha_{t}, \beta\right\rangle=0$. Then we directly obtain $\left(\psi_{s} \psi_{t}\right)\left(e_{\beta}\right)=$ $\left(\psi_{t} \psi_{s}\right)\left(e_{\beta}\right)$.

Case 3: $\left\langle\alpha_{s}, \beta\right\rangle=0$ and $\left\langle\alpha_{s}, \beta\right\rangle=b>0$. Then the equality $\left(\psi_{s} \psi_{t}\right)\left(e_{\beta}\right)=$ $\left(\psi_{t} \psi_{s}\right)\left(e_{\beta}\right)$ is equivalent to

$$
T(s, \beta)=y \cdot T\left(s, \beta-b \alpha_{t}\right)
$$

This equality follows from (D4).

Case $4:\left\langle\alpha_{s}, \beta\right\rangle=0$ and $\left\langle\alpha_{t}, \beta\right\rangle=-b<0$. Then the equality $\left(\psi_{s} \psi_{t}\right)\left(e_{\beta}\right)=$ $\left(\psi_{t} \psi_{s}\right)\left(e_{\beta}\right)$ is equivalent to

$$
T\left(s, \beta+b \alpha_{t}\right)=y \cdot T(s, \beta)
$$

This equality follows from (D4). 
Case 5: $\left\langle\alpha_{s}, \beta\right\rangle=a>0$ and $\left\langle\alpha_{t}, \beta\right\rangle=b>0$. Then the equality $\left(\psi_{s} \psi_{t}\right)\left(e_{\beta}\right)=$ $\left(\psi_{t} \psi_{s}\right)\left(e_{\beta}\right)$ is equivalent to

$$
\begin{aligned}
& T(s, \beta)=y \cdot T\left(s, \beta-b \alpha_{t}\right), \\
& T(t, \beta)=y \cdot T\left(t, \beta-a \alpha_{s}\right) .
\end{aligned}
$$

These two equalities follow from (D3).

Case 6: $\left\langle\alpha_{s}, \beta\right\rangle=a>0$ and $\left\langle\alpha_{t}, \beta\right\rangle=-b<0$. Then the equality $\left(\psi_{s} \psi_{t}\right)\left(e_{\beta}\right)=$ $\left(\psi_{t} \psi_{s}\right)\left(e_{\beta}\right)$ is equivalent to

$$
\begin{aligned}
T\left(s, \beta+b \alpha_{t}\right) & =y \cdot T(s, \beta), \\
T(t, \beta) & =y \cdot T\left(t, \beta-a \alpha_{s}\right) .
\end{aligned}
$$

The first equality follows from (D3) and the second one from (D6).

Case 7: $\left\langle\alpha_{s}, \beta\right\rangle=-a<0$ and $\left\langle\alpha_{t}, \beta\right\rangle=-b<0$. Then the equality $\left(\psi_{s} \psi_{t}\right)\left(e_{\beta}\right)=$ $\left(\psi_{t} \psi_{s}\right)\left(e_{\beta}\right)$ is equivalent to

$$
\begin{aligned}
& T\left(s, \beta+b \alpha_{t}\right)=y \cdot T(s, \beta), \\
& T\left(t, \beta+a \alpha_{s}\right)=y \cdot T(t, \beta) .
\end{aligned}
$$

These two equalities follow from (D6).

Lemma 3.7. Let $s, t \in S$ such that $m_{s, t}=3$. Then $\psi_{s} \psi_{t} \psi_{s}=\psi_{t} \psi_{s} \psi_{t}$.

Proof. Let $\beta \in \Phi^{+}$. We compute $\left(\psi_{s} \psi_{t} \psi_{s}\right)\left(e_{\beta}\right)$ and $\left(\psi_{t} \psi_{s} \psi_{t}\right)\left(e_{\beta}\right)$ replacing $T\left(s, \alpha_{s}\right)$ and $T\left(t, \alpha_{t}\right)$ by $y^{2}$, replacing $T\left(s, \alpha_{t}\right)$ and $T\left(t, \alpha_{s}\right)$ by 0 , and replacing $T\left(s, \alpha_{s}+\alpha_{t}\right)$ and $T\left(t, \alpha_{s}+\alpha_{t}\right)$ by $y^{2}(y-1)$, and we compare both expressions.

Case 1: $\beta=\alpha_{s}$. Then we directly obtain $\left(\psi_{s} \psi_{t} \psi_{s}\right)\left(e_{\beta}\right)=\left(\psi_{t} \psi_{s} \psi_{t}\right)\left(e_{\beta}\right)$.

Case 2: $\beta=\alpha_{s}+\alpha_{t}$. Then we directly obtain $\left(\psi_{s} \psi_{t} \psi_{s}\right)\left(e_{\beta}\right)=\left(\psi_{t} \psi_{s} \psi_{t}\right)\left(e_{\beta}\right)$.

Case $3:\left\langle\alpha_{s}, \beta\right\rangle=0$ and $\left\langle\alpha_{t}, \beta\right\rangle=0$. Then the equality $\left(\psi_{s} \psi_{t} \psi_{s}\right)\left(e_{\beta}\right)=\left(\psi_{t} \psi_{s} \psi_{t}\right)\left(e_{\beta}\right)$ is equivalent to

$$
T(s, \beta)=T(t, \beta) .
$$

This equality follows from Lemma 3.5.

Case $4:\left\langle\alpha_{s}, \beta\right\rangle=0$ and $\left\langle\alpha_{t}, \beta\right\rangle=b>0$. Then the equality $\left(\psi_{s} \psi_{t} \psi_{s}\right)\left(e_{\beta}\right)=$ $\left(\psi_{t} \psi_{s} \psi_{t}\right)\left(e_{\beta}\right)$ is equivalent to

$$
\begin{aligned}
T(t, \beta) & =y \cdot T\left(s, \beta-b \alpha_{t}\right), \\
(1-y) \cdot T(t, \beta)+y \cdot T(s, \beta) & =y^{2} \cdot T\left(t, \beta-b \alpha_{s}-b \alpha_{t}\right) .
\end{aligned}
$$

The first equality follows from (D3) and the second one follows from the first one and from (D5). 
Case 5: $\left\langle\alpha_{s}, \beta\right\rangle=0$ and $\left\langle\alpha_{t}, \beta\right\rangle=-b<0$. Then the equality $\left(\psi_{s} \psi_{t} \psi_{s}\right)\left(e_{\beta}\right)=$ $\left(\psi_{t} \psi_{s} \psi_{t}\right)\left(e_{\beta}\right)$ is equivalent to

$$
\begin{gathered}
(1-y) \cdot T(s, \beta)+T\left(s, \beta+b \alpha_{t}\right)=T(t, \beta), \\
y \cdot T(s, \beta)=(1-y) \cdot T\left(t, \beta+b \alpha_{t}\right)+T\left(t, \beta+b \alpha_{s}+b \alpha_{t}\right) .
\end{gathered}
$$

The first equality follows from (D8) and the second one from (D5).

Case 6: $\left\langle\alpha_{s}, \beta\right\rangle=a>0$ and $\left\langle\alpha_{t}, \beta\right\rangle=b>0$. Then the equality $\left(\psi_{s} \psi_{t} \psi_{s}\right)\left(e_{\beta}\right)=$ $\left(\psi_{t} \psi_{s} \psi_{t}\right)\left(e_{\beta}\right)$ is equivalent to

$$
\begin{aligned}
y \cdot T\left(s, \beta-a \alpha_{s}-(a+b) \alpha_{t}\right) & =(1-y) \cdot T\left(s, \beta-b \alpha_{t}\right)+T(t, \beta), \\
(1-y) \cdot T\left(t, \beta-a \alpha_{s}\right)+T(s, \beta) & =y \cdot T\left(t, \beta-(a+b) \alpha_{s}-b \alpha_{t}\right), \\
T\left(t, \beta-a \alpha_{s}\right) & =T\left(s, \beta+b \alpha_{t}\right) .
\end{aligned}
$$

These three equalities follow from (D3).

Case 7: $\left\langle\alpha_{s}, \beta\right\rangle=a>0,\left\langle\alpha_{t}, \beta\right\rangle=-b<0$, and $a>b$. Then the equality $\left(\psi_{s} \psi_{t} \psi_{s}\right)\left(e_{\beta}\right)=\left(\psi_{t} \psi_{s} \psi_{t}\right)\left(e_{\beta}\right)$ is equivalent to

$$
\begin{aligned}
y^{2} \cdot T\left(s, \beta-a \alpha_{s}-(a-b) \alpha_{t}\right) & =(1-y)^{2} \cdot T(s, \beta)+(1-y) \cdot T\left(s, \beta+b \alpha_{t}\right)+y \cdot T(t, \beta), \\
T(s, \beta) & =T\left(t, \beta-(a-b) \alpha_{s}+b \alpha_{t}\right), \\
y \cdot T\left(t, \beta-a \alpha_{s}\right) & =(1-y) \cdot T(s, \beta)+T\left(s, \beta+b \alpha_{t}\right) .
\end{aligned}
$$

The second and third equalities follow from (D3), and the first one follows from the third one and from (D7).

Case 8: $\left\langle\alpha_{s}, \beta\right\rangle=a>0,\left\langle\alpha_{s}, \beta\right\rangle=-b<0$, and $a=b$. Then the equality $\left(\psi_{s} \psi_{t} \psi_{s}\right)\left(e_{\beta}\right)=\left(\psi_{t} \psi_{s} \psi_{t}\right)\left(e_{\beta}\right)$ is equivalent to

$$
\begin{aligned}
y \cdot T\left(s, \beta-a \alpha_{s}\right) & =(1-y)^{2} \cdot T(s, \beta)+(1-y) \cdot T\left(s, \beta+a \alpha_{t}\right)+y \cdot T(t, \beta), \\
T\left(t, \beta+a \alpha_{t}\right) & =y \cdot T(s, \beta), \\
y \cdot T\left(t, \beta-a \alpha_{s}\right) & =(1-y) \cdot T(s, \beta)+T\left(s, \beta+a \alpha_{t}\right) .
\end{aligned}
$$

The second equality follows from (D3), the third one follows from (D5), and the first one follows from the third one and from (D8).

Case 9: $\left\langle\alpha_{s}, \beta\right\rangle=a>0,\left\langle\alpha_{t}, \beta\right\rangle=-b<0$, and $a<b$. Then the equality $\left(\psi_{s} \psi_{t} \psi_{s}\right)\left(e_{\beta}\right)=\left(\psi_{t} \psi_{s} \psi_{t}\right)\left(e_{\beta}\right)$ is equivalent to

$$
\begin{aligned}
y(1-y) \cdot T\left(s, \beta-a \alpha_{s}\right) & +y \cdot T\left(s, \beta-a \alpha_{s}+(b-a) \alpha_{t}\right) \\
= & (1-y)^{2} \cdot T(s, \beta)+(1-y) \cdot T\left(s, \beta+b \alpha_{t}\right)+y \cdot T(t, \beta), \\
y \cdot T(s, \beta)= & (1-y) \cdot T\left(t, \beta+b \alpha_{t}\right)+T\left(t, \beta+(b-a) \alpha_{s}+b \alpha_{t}\right), \\
y \cdot T\left(t, \beta-a \alpha_{s}\right)= & (1-y) \cdot T(s, \beta)+T\left(s, \beta+b \alpha_{t}\right) .
\end{aligned}
$$


The second equality follows from (D3), the third one follows from (D7), and the first one follows from the third one and from (D9).

Case 10: $\left\langle\alpha_{s}, \beta\right\rangle=-a<0$ and $\left\langle\alpha_{t}, \beta\right\rangle=-b<0$. Then the equality $\left(\psi_{s} \psi_{t} \psi_{s}\right)\left(e_{\beta}\right)=$ $\left(\psi_{t} \psi_{s} \psi_{t}\right)\left(e_{\beta}\right)$ is equivalent to

$$
\begin{aligned}
& (1-y) \cdot T\left(s, \beta+a \alpha_{s}\right)+T\left(s, \beta+a \alpha_{s}+(a+b) \alpha_{t}\right)=y \cdot T(t, \beta), \\
& y \cdot T(s, \beta)=(1-y) \cdot T\left(t, \beta+b \alpha_{t}\right)+T\left(t, \beta+(a+b) \alpha_{s}+b \alpha_{t}\right), \\
& (1-y) \cdot T(t, \beta)+T\left(t, \beta+a \alpha_{s}\right)=(1-y) \cdot T(s, \beta)+T\left(s, \beta+b \alpha_{t}\right) .
\end{aligned}
$$

The first and second equalities follow from (D7), and the third one follows from (D9).

Lemma 3.8. Let $s \in S$. Then $\psi_{s}$ is invertible.

Proof. Let $\rho_{s}: V \rightarrow V$ be the linear transformation defined by

$$
\rho_{s}\left(e_{\beta}\right)= \begin{cases}x^{-1} y^{-2} \cdot e_{\alpha_{s}} & \text { if } \beta=\alpha_{s} \\ e_{\beta}-y^{-2} T(s, \beta) \cdot e_{\alpha_{s}} & \text { if }\left\langle\alpha_{s}, \beta\right\rangle=0 \\ \left(1-y^{-1}\right) \cdot e_{\beta}+e_{\beta-a \alpha_{s}} & \\ \quad-y^{-2} T\left(s, \beta-a \alpha_{s}\right) \cdot e_{\alpha_{s}} & \\ \quad+y^{-2}\left(y^{-1}-1\right) T(s, \beta) \cdot e_{\alpha_{s}} & \text { if }\left\langle\alpha_{s}, \beta\right\rangle=a>0 \text { and } \beta \neq \alpha_{s} \\ y^{-1} \cdot e_{\beta+a \alpha_{s}}-y^{-3} T\left(s, \beta+a \alpha_{s}\right) \cdot e_{\alpha_{s}} & \text { if }\left\langle\alpha_{s}, \beta\right\rangle=-a<0\end{cases}
$$

A direct case by case calculation shows that $\psi_{s} \circ \rho_{s}=\rho_{s} \circ \psi_{s}=\operatorname{Id}_{V}$. So, $\psi_{s}$ is invertible.

This finishes the proof of Theorem 3.2.

\section{Faithfulness}

Throughout this section, $\Gamma$ is again assumed to be a Coxeter graph of small type with no triangle. Our goal here is to prove the following.

Theorem 4.1. The representation $\psi: G_{\Gamma}^{+} \rightarrow \mathrm{Gl}(V)$ defined in Section 3 is faithful.

Since $\mathrm{Gl}(V)$ is a group, it follows:

Corollary 4.2. The homomorphism $\iota: G_{\Gamma}^{+} \rightarrow G_{\Gamma}$ is injective. 
Let $V_{+}=\oplus_{\beta \in \Phi^{+}} \mathbf{Q}[x, y] e_{\beta}$ denote the free $\mathbf{Q}[x, y]$-module having $\mathcal{E}=\left\{e_{\beta} ; \beta \in\right.$ $\left.\Phi^{+}\right\}$as a basis. The coefficients of $\psi(g)$ lie in $\mathbf{Q}[x, y]$, for all $g \in G_{\Gamma}^{+}$, thus $V_{+}$is invariant by the action of $G_{\Gamma}^{+}$. We denote by $\psi_{+}: G_{\Gamma}^{+} \rightarrow \operatorname{End}\left(V_{+}\right)$the restriction of $\psi$ to $V_{+}$.

Let $V_{0}=\oplus_{\beta \in \Phi^{+}} \mathbf{R} e_{\beta}$ denote the real vector space having $\mathcal{E}$ as a basis. Replacing $x$ by 0 and $y$ by a value $0<y_{0}<1$, the homomorphism $\psi_{+}$induces a homomorphism $\psi_{0}: G_{\Gamma}^{+} \rightarrow \operatorname{End}\left(V_{0}\right)$.

Let $\mathcal{H}$ be the vector space of formal series $\sum_{\beta \in \Phi^{+}} \lambda_{\beta} e_{\beta}$, and let $\mathcal{L}(\mathcal{H})$ be the space of linear transformations of $\mathcal{H}$. Observe that $\psi_{0}\left(\sigma_{s}\right)$ defines a linear transformation $\psi_{\infty}\left(\sigma_{s}\right)$ of $\mathcal{H}$, because each row has only finitely many non-zero entries, thus $\psi_{0}: G_{\Gamma}^{+} \rightarrow \operatorname{End}\left(V_{0}\right)$ induces a homomorphism $\psi_{\infty}: G_{\Gamma}^{+} \rightarrow \mathcal{L}(\mathcal{H})$.

Definition. Let $A$ be a subset of $\Phi^{+}$. Then $U_{A}$ denotes the set of series $\sum \lambda_{\beta} e_{\beta} \in$ $\mathcal{H}$ such that:

- $\lambda_{\beta} \geq 0$ for all $\beta \in \Phi^{+}$;

- $\lambda_{\beta}=0$ if and only if $\beta \in A$.

Note that $U_{A}$ is nonempty, even if $\Phi^{+} \backslash A$ is infinite, and one has $U_{A} \cap U_{B}=\emptyset$ if $A \neq B$.

Lemma 4.3. Let $A \subset \Phi^{+}$and $g \in G_{\Gamma}^{+}$. There exists a unique subset $B \subset \Phi^{+}$ such that $\psi_{\infty}(g) \cdot U_{A} \subset U_{B}$.

Proof. The hypothesis $0<y_{0}<1$ implies that the coefficients of $\psi_{\infty}\left(\sigma_{s}\right)$ are $\geq 0$ for all $s \in S$, thus the coefficients of $\psi_{\infty}(g)$ are $\geq 0$. Let $\psi_{\infty}(g)\left(e_{\beta}\right)=\sum_{\gamma \in \Phi^{+}} a_{\gamma, \beta} e_{\gamma}$, and let $\operatorname{Supp}_{g}\left(e_{\beta}\right)$ denote the set of $\gamma \in \Phi^{+}$such that $a_{\gamma, \beta}>0$. Let

$$
A^{\prime}=\Phi^{+} \backslash A, \quad B^{\prime}=\cup_{\beta \in A^{\prime}} \operatorname{Supp}_{g}\left(e_{\beta}\right), \quad B=\Phi^{+} \backslash B^{\prime} .
$$

Then $\psi_{\infty}(g) \cdot U_{A} \subset U_{B}$.

Definition. Let $A \subset \Phi^{+}$and $g \in G_{\Gamma}^{+}$. Then $g * A=B$ denotes the unique subset $B \subset \Phi^{+}$such that $\psi_{\infty}(g) \cdot U_{A} \subset U_{B}$.

Lemma 4.4. Let $A \subset \Phi^{+}$and $s \in S$. Then

$$
\begin{aligned}
\sigma_{s} * A=\left\{\alpha_{s}\right\} & \cup\left\{\beta \in \Phi^{+} ;\left\langle\alpha_{s}, \beta\right\rangle=0 \text { and } \beta \in A\right\} \\
\cup\left\{\beta \in \Phi^{+} ;\left\langle\alpha_{s}, \beta\right\rangle\right. & \left.=a>0, \beta \neq \alpha_{s}, \text { and } \beta-a \alpha_{s} \in A\right\} \\
& \cup\left\{\beta \in \Phi^{+} ;\left\langle\alpha_{s}, \beta\right\rangle=-a<0, \beta \in A, \text { and } \beta+a \alpha_{s} \in A\right\}
\end{aligned}
$$


Proof. Let $\beta \in \Phi^{+}$. Then

$$
\operatorname{Supp}_{\sigma_{s}}\left(e_{\beta}\right)= \begin{cases}\emptyset & \text { if } \beta=\alpha_{s}, \\ \{\beta\} & \text { if }\left\langle\alpha_{s}, \beta\right\rangle=0 \\ \left\{\beta-a \alpha_{s}\right\} & \text { if }\left\langle\alpha_{s}, \beta\right\rangle=a>0 \text { and } \beta \neq \alpha_{s}, \\ \left\{\beta, \beta+a \alpha_{s}\right\} & \text { if }\left\langle\alpha_{s}, \beta\right\rangle=-a<0 .\end{cases}
$$

Let $A^{\prime}=\Phi^{+} \backslash A$ and $B^{\prime}=\cup_{\beta \in A^{\prime}} \operatorname{Supp}_{\sigma_{s}}\left(e_{\beta}\right)$. Then

$$
\begin{aligned}
& B^{\prime}=\left\{\beta \in \Phi^{+} ;\left\langle\alpha_{s}, \beta\right\rangle=0 \text { and } \beta \in A^{\prime}\right\} \\
& \cup\left\{\beta \in \Phi^{+} ;\left\langle\alpha_{s}, \beta\right\rangle=a>0, \text { and } \beta-a \alpha_{s} \in A^{\prime}\right\} \\
& \cup\left\{\beta \in \Phi^{+} ;\left\langle\alpha_{s}, \beta\right\rangle=-a<0, \text { and either } \beta \in A^{\prime} \text { or } \beta+a \alpha_{s} \in A^{\prime}\right\}
\end{aligned}
$$

thus

$$
\begin{aligned}
\sigma_{s} * A=B= & \Phi^{+} \backslash B^{\prime} \\
=\left\{\alpha_{s}\right\} & \cup\left\{\beta \in \Phi^{+} ;\left\langle\alpha_{s}, \beta\right\rangle=0 \text { and } \beta \in A\right\} \\
& \cup\left\{\beta \in \Phi^{+} ;\left\langle\alpha_{s}, \beta\right\rangle=a>0, \text { and } \beta-a \alpha_{s} \in A\right\} \\
& \cup\left\{\beta \in \Phi^{+} ;\left\langle\alpha_{s}, \beta\right\rangle=-a<0, \beta \in A, \text { and } \beta+a \alpha_{s} \in A\right\} .
\end{aligned}
$$

Remark. Let $A \subset \Phi^{+}$and $s \in S$. Then

$$
\begin{aligned}
& s\left(A \backslash\left\{\alpha_{s}\right\}\right)=\left\{\beta \in \Phi^{+} ;\left\langle\alpha_{s}, \beta\right\rangle=0 \text { and } \beta \in A\right\} \\
& \cup\left\{\beta \in \Phi^{+} ;\left\langle\alpha_{s}, \beta\right\rangle=a>0, \text { and } \beta-a \alpha_{s} \in A\right\} \\
& \cup\left\{\beta \in \Phi^{+} ;\left\langle\alpha_{s}, \beta\right\rangle=-a<0 \text { and } \beta+a \alpha_{s} \in A\right\} .
\end{aligned}
$$

In particular, one has

$$
\sigma_{s} * A \subset\left\{\alpha_{s}\right\} \cup s\left(A \backslash\left\{\alpha_{s}\right\}\right) .
$$

Lemma 4.5. Let $A$ be a closed subset of $\Phi^{+}$and $s \in S$. Then $\sigma_{s} * A$ is also a closed subset.

Proof. Since $A$ is finite, $\sigma_{s} * A$ is also finite. Now, we take $\beta_{1}, \beta_{2} \in \sigma_{s} * A$ and we prove:

- $\left\langle\beta_{1}, \beta_{2}\right\rangle \geq-1$

- if $\left\langle\beta_{1}, \beta_{2}\right\rangle=-1$, then $\beta_{1}+\beta_{2} \in \sigma_{s} * A$.

Assume first that $\beta_{1}=\alpha_{s}$. If $\left\langle\alpha_{s}, \beta_{2}\right\rangle=-a<0$, then $\beta_{2}, \beta_{2}+a \alpha_{s} \in A$ (by Lemma 4.4). The fact that $A$ is closed implies

$$
\left\langle\beta_{2}, \beta_{2}+a \alpha_{s}\right\rangle=\left\langle\beta_{2}, \beta_{2}\right\rangle+a\left\langle\beta_{2}, \alpha_{s}\right\rangle=2-a^{2} \geq-1,
$$


and this inequality holds only if $a=1$. This shows that $\left\langle\alpha_{s}, \beta_{2}\right\rangle \geq-1$. Suppose $\left\langle\alpha_{s}, \beta_{2}\right\rangle=-1$. Then $\left\langle\alpha_{s}, \beta_{2}+\alpha_{s}\right\rangle=1$ and $\beta_{2} \in A$ (by Lemma 4.4), thus, by Lemma 4.4, $\beta_{2}+\alpha_{s} \in \sigma_{s} * A$.

Assume now that $\beta_{1} \neq \alpha_{s}$ and $\beta_{2} \neq \alpha_{s}$. One has $\beta_{1}, \beta_{2} \in s\left(A \backslash\left\{\alpha_{s}\right\}\right)$, thus $s\left(\beta_{1}\right), s\left(\beta_{2}\right) \in A$, therefore $\left\langle\beta_{1}, \beta_{2}\right\rangle=\left\langle s\left(\beta_{1}\right), s\left(\beta_{2}\right)\right\rangle \geq-1$ (since $A$ is closed). Suppose now that $\left\langle\beta_{1}, \beta_{2}\right\rangle=-1$.

Case 1: $\left\langle\alpha_{s}, \beta_{1}\right\rangle=0$ and $\left\langle\alpha_{s}, \beta_{2}\right\rangle=0$. Then $\left\langle\alpha_{s}, \beta_{1}+\beta_{2}\right\rangle=0$. Moreover, one has $\beta_{1}, \beta_{2} \in A$ (by Lemma 4.4), thus $\beta_{1}+\beta_{2} \in A$ (since $A$ is closed), therefore $\beta_{1}+\beta_{2} \in \sigma_{s} * A$ (by Lemma 4.4).

Case 2: $\left\langle\alpha_{s}, \beta_{1}\right\rangle=0$ and $\left\langle\alpha_{s}, \beta_{2}\right\rangle=b>0$. Then $\left\langle\alpha_{s}, \beta_{1}+\beta_{2}\right\rangle=b>0$. Moreover, one has $\beta_{1}, \beta_{2}-b \alpha_{s} \in A$ (by Lemma 4.4) and $\left\langle\beta_{1}, \beta_{2}-b \alpha_{s}\right\rangle=-1$, thus $\beta_{1}+\beta_{2}-$ $b \alpha_{s} \in A$ (since $A$ is closed), therefore $\beta_{1}+\beta_{2} \in \sigma_{s} * A$ (by Lemma 4.4).

Case 3: $\left\langle\alpha_{s}, \beta_{1}\right\rangle=0$ and $\left\langle\alpha_{s}, \beta_{2}\right\rangle=-b<0$. Then $\left\langle\alpha_{s}, \beta_{1}+\beta_{2}\right\rangle=-b<0$. Moreover, one has $\beta_{1}, \beta_{2}, \beta_{2}+b \alpha_{s} \in A$ (by Lemma 4.4) and $\left\langle\beta_{1}, \beta_{2}\right\rangle=\left\langle\beta_{1}, \beta_{2}+\right.$ $\left.b \alpha_{s}\right\rangle=-1$, thus $\beta_{1}+\beta_{2}, \beta_{1}+\beta_{2}+b \alpha_{s} \in A$ (since $A$ is closed), therefore $\beta_{1}+\beta_{2} \in$ $\sigma_{s} * A$ (by Lemma 4.4).

Case 4: $\left\langle\alpha_{s}, \beta_{1}\right\rangle=a>0$ and $\left\langle\alpha_{s}, \beta_{2}\right\rangle=b>0$. Then $\left\langle\alpha_{s}, \beta_{1}+\beta_{2}\right\rangle=a+b>0$. Moreover, one has $\beta_{1}-a \alpha_{s}, \beta_{2}-b \alpha_{s} \in A$ (by Lemma 4.4) and $\left\langle\beta_{1}-a \alpha_{s}, \beta_{2}-b \alpha_{s}\right\rangle=$ -1 , thus $\beta_{1}+\beta_{2}-(a+b) \alpha_{s} \in A$ (since $A$ is closed), therefore $\beta_{1}+\beta_{2} \in \sigma_{s} * A$ (by Lemma 4.4).

Case 5: $\left\langle\alpha_{s}, \beta_{1}\right\rangle=a>0$ and $\left\langle\alpha_{s}, \beta_{2}\right\rangle=-b<0$. Note first that $\beta_{2}, \beta_{2}+b \alpha_{s} \in A$ (by Lemma 4.4), thus $\left\langle\beta_{2}, \beta_{2}+b \alpha_{s}\right\rangle=2-b^{2} \geq-1$ (since $A$ is closed), therefore $b=1$.

Suppose $a=1$. Then $\left\langle\alpha_{s}, \beta_{1}+\beta_{2}\right\rangle=0$. One has $\beta_{1}-\alpha_{s}, \beta_{2}+\alpha_{s} \in A$ (by Lemma 4.4) and $\left\langle\beta_{1}-\alpha_{s}, \beta_{2}+\alpha_{s}\right\rangle=-1$, thus $\beta_{1}+\beta_{2} \in A$ (since $A$ is closed), therefore $\beta_{1}+\beta_{2} \in \sigma_{s} * A$ (by Lemma 4.4).

Suppose $a \geq 2$. Then $\left\langle\alpha_{s}, \beta_{1}+\beta_{2}\right\rangle=a-1>0$. One has $\beta_{1}-a \alpha_{s}, \beta_{2}+\alpha_{s} \in A$ (by Lemma 4.4) and $\left\langle\beta_{1}-a \alpha_{s}, \beta_{2}+\alpha_{s}\right\rangle=-1$, thus $\beta_{1}+\beta_{2}-(a-1) \alpha_{s} \in A$ (since $A$ is closed), therefore $\beta_{1}+\beta_{2} \in \sigma_{s} * A$ (by Lemma 4.4).

Case 6: $\left\langle\alpha_{s}, \beta_{1}\right\rangle=-a<0$ and $\left\langle\alpha_{s}, \beta_{2}\right\rangle=-b<0$. Then $\beta_{1}, \beta_{2}+b \alpha_{s} \in A$ (by Lemma 4.4) and $\left\langle\beta_{1}, \beta_{2}+b \alpha_{s}\right\rangle=-1-a b<-1$. This contradicts the definition of a closed subset, thus this case does not hold.

Corollary 4.6. Let $A$ be a closed subset of $\Phi^{+}$and $g \in G_{\Gamma}^{+}$. Then $g * A$ is also a closed subset.

Lemma 4.7. Let $w \in W$ and $s \in S$ such that $l(s w)=l(w)-1$, and let $A$ be a closed subset of $\Phi^{+}$. One has $\Phi_{w} \subset\left\{\alpha_{s}\right\} \cup s\left(A \backslash\left\{\alpha_{s}\right\}\right)$ if and only if $w<$ $L\left(\sigma_{s} \cdot(\tau \circ C)(A)\right)$.

Proof. The equality $l(s w)=l(w)-1$ implies, by Proposition 2.4, that $\Phi_{w}=$ $\left\{\alpha_{s}\right\} \sqcup s \cdot \Phi_{s w}$. So, the inclusion $\Phi_{w} \subset\left\{\alpha_{s}\right\} \cup s\left(A \backslash\left\{\alpha_{s}\right\}\right)$ is equivalent to $s\left(\Phi_{s w}\right) \subset$ 
$s\left(A \backslash\left\{\alpha_{s}\right\}\right)$, which is equivalent to $\Phi_{s w} \subset A$ (we cannot have $\alpha_{s} \in \Phi_{s w}$ because $l(s w)<l(w))$. This inclusion is equivalent to $s w<C(A)$, which is equivalent to $\tau(s w)<(\tau \circ C)(A)$, which is equivalent to $\sigma_{s} \cdot \tau(s w)=\tau(w)<\sigma_{s} \cdot(\tau \circ C)(A)$, which is equivalent to $w<L\left(\sigma_{s} \cdot(\tau \circ C)(A)\right)$.

Lemma 4.8. Let $A$ and $B$ be two closed subsets of $\Phi^{+}$and $s \in S$. If $\left\{\alpha_{s}\right\} \subset B \subset$ $\left\{\alpha_{s}\right\} \cup s\left(A \backslash\left\{\alpha_{s}\right\}\right)$, then $B \subset \sigma_{s} * A$.

Proof. By Lemma 4.4 and the remark preceding Lemma 4.5, it suffices to show that: if $\beta \in B$ is such that $\left\langle\alpha_{s}, \beta\right\rangle=-a<0$, then $\beta \in A$. One has $\alpha_{s}, \beta \in B$ and $B$ is a closed subset, thus $\left\langle\alpha_{s}, \beta\right\rangle=-1$ and $\beta+\alpha_{s} \in B$. It follows that $s(\beta)=\alpha_{s}+\beta \in B \backslash\left\{\alpha_{s}\right\} \subset s\left(A \backslash\left\{\alpha_{s}\right\}\right)$, thus $\beta \in A$.

Lemma 4.9. Let $A$ be a closed subset of $\Phi^{+}$and $g \in G_{\Gamma}^{+}$. Then

$$
C(g * A)=L(g \cdot(\tau \circ C)(A)) .
$$

Proof. We argue by induction on $l(g)$. Assume first that $l(g)=1$. Then $g=\sigma_{s}$ for some $s \in S$. Let $w_{1}=C\left(\sigma_{s} * A\right)$ and $w_{2}=L\left(\sigma_{s} \cdot(\tau \circ C)(A)\right)$. Since $\alpha_{s} \in \sigma_{s} * A$, one has $\Phi_{s}=\left\{\alpha_{s}\right\} \subset \sigma_{s} * A$, thus $s<w_{1}$, namely, $l\left(s w_{1}\right)=l\left(w_{1}\right)-1$. Moreover, $\Phi_{w_{1}} \subset \sigma_{s} * A \subset\left\{\alpha_{s}\right\} \cup s\left(A \backslash\left\{\alpha_{s}\right\}\right)$, thus, by Lemma 4.7, $w_{1}<w_{2}$. One has $\tau(s)=\sigma_{s}<\sigma_{s} \cdot(\tau \circ C)(A)$, thus $s<w_{2}$, namely, $l\left(s w_{2}\right)=l\left(w_{2}\right)-1$. By Lemma 4.7, it follows that $\left\{\alpha_{s}\right\} \subset \Phi_{w_{2}} \subset\left\{\alpha_{s}\right\} \cup s\left(A \backslash\left\{\alpha_{s}\right\}\right)$ and so, by Lemma 4.8, $\Phi_{w_{2}} \subset \sigma_{s} * A$. This implies that $w_{2}<w_{1}$.

Assume now that $l(g) \geq 2$. We write $g=\sigma_{s} g_{1}$ where $s \in S$ and $l\left(g_{1}\right)=l(g)-1$. Then, by induction and by Proposition 2.3,

$$
\begin{aligned}
C(g * A) & =C\left(\sigma_{s} *\left(g_{1} * A\right)\right)=L\left(\sigma_{s}(\tau \circ C)\left(g_{1} * A\right)\right) \\
& =L\left(\sigma_{s}(\tau \circ L)\left(g_{1}(\tau \circ C)(A)\right)\right)=L(g(\tau \circ C)(A)) .
\end{aligned}
$$

Definition. Let $\mathcal{C}$ denote the set of closed subsets of $\Phi^{+}$. For $w \in W$ we set

$$
\mathcal{U}_{w}=\bigcup_{A \in \mathcal{C}, C(A)=w} U_{A} .
$$

Note that $\mathcal{U}_{w} \neq \emptyset$ (since it contains $U_{\Phi_{w}}$ ), and one has $\mathcal{U}_{u} \cap \mathcal{U}_{v}=\emptyset$ if $u \neq v$.

Lemma 4.10. Let $g \in G_{\Gamma}^{+}$and $w \in W$. Then

$$
\psi_{\infty}(g) \cdot \mathcal{U}_{w} \subset \mathcal{U}_{L(g \cdot \tau(w))}
$$

Proof. Let $A \in \mathcal{C}$ such that $C(A)=w$. One has $\psi_{\infty}(g) \cdot U_{A} \subset U_{g * A}$, and, by Lemma $4.9, C(g * A)=L(g \cdot(\tau \circ C)(A))=L(g \cdot \tau(w))$, thus $\psi_{\infty}(g) \cdot U_{A} \subset \mathcal{U}_{L(g \cdot \tau(w))}$. This shows that $\psi_{\infty}(g) \cdot \mathcal{U}_{w} \subset \mathcal{U}_{L(g \cdot \tau(w))}$. 
Proof of Theorem 4.1. Let $f, g \in G_{\Gamma}^{+}$such that $\psi(f)=\psi(g)$. We write $f=\tau(u) f_{1}$ and $g=\tau(v) g_{2}$, where $u=L(f), v=L(g)$, and $f_{1}, g_{1} \in G_{\Gamma}^{+}$. Note that $u=1$ if and only if $f=1$, and $v=1$ if and only if $g=1$. Lemma 4.10 implies that $\psi_{\infty}(f) \cdot \mathcal{U}_{1} \subset \mathcal{U}_{L(f)}=\mathcal{U}_{u}$, and that $\psi_{\infty}(g) \cdot \mathcal{U}_{1} \subset \mathcal{U}_{L(g)}=\mathcal{U}_{v}$. Since $\psi_{\infty}(f)=\psi_{\infty}(g)$, and since $\mathcal{U}_{u} \cap \mathcal{U}_{v}=\emptyset$ if $u \neq v$, it follows that $u=v$.

We prove now that $f=g$ by induction on $l(f)$. If $l(f)=0$, then $f=1$, thus $u=v=1$, therefore $g=1$. Suppose $l(f)>0$. Then $l\left(f_{1}\right)<l(f)$ and

$$
\psi\left(f_{1}\right)=\psi(\tau(u))^{-1} \psi(f)=\psi(\tau(v))^{-1} \psi(g)=\psi\left(g_{1}\right) .
$$

By the inductive hypothesis, it follows that $f_{1}=g_{1}$, thus $f=\tau(u) f_{1}=\tau(v) g_{1}=g$.

\section{The general case}

Now, we assume that $\Gamma$ is any Coxeter graph. The goal of this section is to prove the following.

Theorem 5.1. There exists an injective homomorphism $\phi: G_{\Gamma}^{+} \rightarrow G_{\tilde{\Gamma}}^{+}$from $G_{\Gamma}^{+}$ to an Artin monoid $G_{\tilde{\Gamma}}^{+}$associated to a Coxeter graph $\tilde{\Gamma}$ of small type with no triangle.

Since we already know by Corollary 4.2 that $\iota: G_{\tilde{\Gamma}}^{+} \rightarrow G_{\tilde{\Gamma}}$ is injective, Theorem 5.1 finishes the proof of Theorem 1.1.

We start summarizing some well known properties of $G_{\Gamma}^{+}$that can be found in [BS] and [Mic].

We say that $g \in G_{\Gamma}^{+}$is a common multiple of a finite subset $F=\left\{f_{1}, \ldots, f_{n}\right\} \subset$ $G_{\Gamma}^{+}$if $f_{i}<g$ for all $i=1, \ldots, n$. If $F=\left\{f_{1}, \ldots, f_{n}\right\}$ has a common multiple, then it has a least common multiple, which is obviously unique, and which will be denoted by $f_{1} \vee \cdots \vee f_{n}$.

Let $s, t \in S$. The subset $\left\{\sigma_{s}, \sigma_{t}\right\}$ has a common multiple if and only if $m_{s, t}<$ $+\infty$. In that case, one has $\sigma_{s} \vee \sigma_{t}=\operatorname{prod}\left(\sigma_{s}, \sigma_{t} ; m_{s, t}\right)$. More generally, for a subset $T \subset S$, the set $\Sigma_{T}=\left\{\sigma_{t} ; t \in T\right\}$ has a common multiple if and only if the subgroup $W_{T}$ of $W$ generated by $T$ is finite. In that case, the least common multiple of $\Sigma_{T}$ is denoted by $\Delta_{T}$. It is equal to $\tau\left(w_{T}\right)$, where $w_{T}$ denotes the element of maximal length in $W_{T}$. If $W$ is finite, namely, if $\Gamma$ is of spherical type, then we will denote by $\Delta=\Delta(\Gamma)$ the least common multiple of $\Sigma=\left\{\sigma_{s} ; s \in S\right\}$.

Let $T \subset S$ and $f, g \in G_{\Gamma}^{+}$. If $f$ and $g$ have a common multiple and both lie in the submonoid generated by $\Sigma_{T}=\left\{\sigma_{t} ; t \in T\right\}$, then $f \vee g$ also lies in this submonoid.

Definition. Let $\Gamma$ and $\Gamma^{\prime}$ be two Coxeter graphs, let $S$ be the set of vertices of $\Gamma$, and let $\phi: G_{\Gamma}^{+} \rightarrow G_{\Gamma^{\prime}}^{+}$be a homomorphism. We say that $\phi$ respects $l \mathrm{~cm}$ 's if 
- $\phi\left(\sigma_{s}\right) \neq 1$ for all $s \in S$;

- $\left\{\phi\left(\sigma_{s}\right), \phi\left(\sigma_{t}\right)\right\}$ has a common multiple if and only if $m_{s, t}<+\infty$;

- if $m_{s, t}<+\infty$, then $\phi\left(\sigma_{s} \vee \sigma_{t}\right)=\phi\left(\sigma_{s}\right) \vee \phi\left(\sigma_{t}\right)$.

The following theorem can be found in [Cri, Thm. 1.3].

Theorem 5.2 (Crisp). If a homomorphism $\phi: G_{\Gamma}^{+} \rightarrow G_{\Gamma^{\prime}}^{+}$between Artin monoids respects lcm's, then it is injective.

Proof of Theorem 5.1. Let $A_{n}$ be the Coxeter graph of Figure 1. Let $f, g$ be the elements of $G_{A_{n}}^{+}$defined by $f=\sigma_{1} \sigma_{3} \sigma_{5} \ldots$ and $g=\sigma_{2} \sigma_{4} \sigma_{6} \ldots$ It is shown in [BS, Lem. 5.8] that

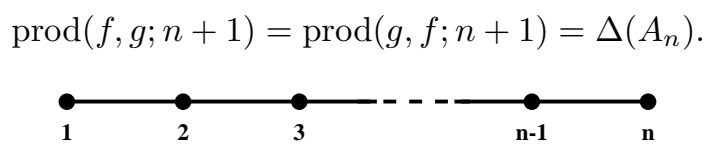

Figure 1. The Coxeter graph $A_{n}$

Let $m \geq 3$, and let $\Gamma(m)$ denote the Coxeter graph illustrated in Figure 2 . It is a bipartite graph whose set of vertices is the disjoint union $I \sqcup J$, where $|I|=|J|=m-1$. As a Coxeter graph, $\Gamma(m)$ is the disjoint union of two copies of $A_{m-1}$. Let $f, g$ be the elements of $G_{\Gamma(m)}^{+}$defined by $f=\prod_{i \in I} \sigma_{i}$ and $g=\prod_{j \in J} \sigma_{j}$. Then, by (1), one has

$$
\operatorname{prod}(f, g ; m)=\operatorname{prod}(g, f ; m)=\Delta(\Gamma(m)) .
$$

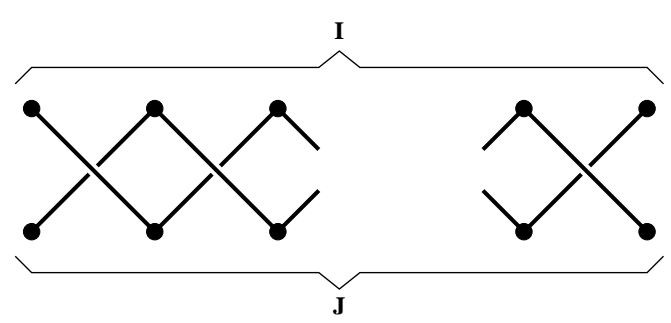

Figure 2. The Coxeter graph $\Gamma(m)$

Let $k \in \mathbf{N}$. We denote by $k \Gamma(m)$ the disjoint union of $k$ copies of $\Gamma(m)$. It is a bipartite graph whose set of vertices is the disjoint union $k I \sqcup k J$, where $k I$ denotes the disjoint union of $k$ copies of $I$, and $k J$ denotes the disjoint union of $k$ copies of $J$. Let $f, g$ be the elements of $G_{k \Gamma(m)}^{+}$defined by $f=\prod_{i \in k I} \sigma_{i}$ and $g=\prod_{j \in k J} \sigma_{j}$. Then, by (2), one has

$$
\operatorname{prod}(f, g ; m)=\operatorname{prod}(g, f ; m)=\Delta(k \Gamma(m)) .
$$


Let $\Gamma(\infty)$ denote the Coxeter graph illustrated in Figure 3. It is bipartite graph whose set of vertices is the disjoint union $I \sqcup J$, where $I=\left\{i_{1}, i_{2}\right\}$ and $J=\left\{j_{1}, j_{2}\right\}$. Let $f, g$ be the elements of $G_{\Gamma(\infty)}^{+}$defined by $f=\sigma_{i_{1}} \sigma_{i_{2}}$ and $g=\sigma_{j_{1}} \sigma_{j_{2}}$. A common multiple of $f$ and $g$ would be a common multiple of $\left\{\sigma_{i_{1}}, \sigma_{i_{2}}, \sigma_{j_{1}}, \sigma_{j_{2}}\right\}$. But $\Gamma(\infty)$ is not of spherical type, thus such a common multiple does not exist. So, $f$ and $g$ have no common multiple.

Let $k \in \mathbf{N}$. We denote by $k \Gamma(\infty)$ the disjoint union of $k$ copies of $\Gamma(\infty)$. It is a bipartite graph whose set of vertices is the disjoint union $k I \sqcup k J$, where $k I$ denotes the disjoint union of $k$ copies of $I$, and $k J$ denotes the disjoint union of $k$ copies of $J$. Let $f$ and $g$ be the elements of $G_{k \Gamma(\infty)}^{+}$defined by $f=\prod_{i \in k I} \sigma_{i}$ and $g=\prod_{j \in k J} \sigma_{j}$. Then, as before, $f$ and $g$ have no common multiple.

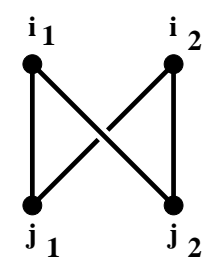

Figure 3. The Coxeter graph $\Gamma(\infty)$

Now, let $\Gamma$ be any Coxeter graph. Let $N$ be the least common multiple of $\left\{m_{s, t}-1 ; s, t \in S, s \neq t, m_{s, t}<+\infty\right\}$. For all $s \in S$ we take an abstract set $I(s)$ with $2 N$ elements. We construct a Coxeter graph of small type $\Gamma^{\prime}$ as follows.

- The set of vertices of $\Gamma^{\prime}$ is the disjoint union of the $I(s), s \in S$.

- If $m_{s, t}=2$, then there is no edge joining two vertices in $I(s) \sqcup I(t)$.

- If $3 \leq m_{s, t}<+\infty$, then the full subgraph of $\Gamma^{\prime}$ generated by $I(s) \sqcup I(t)$ is isomorphic to $\left(\frac{2 N}{m_{s, t}-1}\right) \Gamma\left(m_{s, t}\right)$ with an isomorphism which takes $I(s)$ to $\left(\frac{2 N}{m_{s, t}-1}\right) I$ and $I(t)$ to $\left(\frac{2 N}{m_{s, t}-1}\right) J$.

- If $m_{s, t}=+\infty$, then the full subgraph of $\Gamma^{\prime}$ generated by $I(s) \sqcup I(t)$ is isomorphic to $N \Gamma(\infty)$ with an isomorphism which takes $I(s)$ to $N I$ and $I(t)$ to NJ.

Such a graph always exists but is not unique in general. By the above considerations, there is a well defined homomorphism $\phi: G_{\Gamma}^{+} \rightarrow G_{\Gamma^{\prime}}^{+}$which sends $\sigma_{s}$ to $\prod_{i \in I(s)} \sigma_{i}$ for all $s \in S$, and this homomorphism respects lcm's, so, is injective by Theorem 5.2. Note also that: if $\Gamma$ is of small type, then one can choose $N=1$ rather than the above $N=2$, and at least one of the $\Gamma^{\prime}$ thus obtained is bipartite, and therefore has no triangle. So, applying twice the above construction, one gets a Coxeter graph $\tilde{\Gamma}$ of small type with no triangle and a monomorphism $\phi: G_{\Gamma}^{+} \rightarrow G_{\tilde{\Gamma}}^{+}$. 


\section{Two lemmas}

Lemma 3.3. Let $s \in S$ and $\beta \in \Phi^{+}$such that $\left\langle\alpha_{s}, \beta\right\rangle=0$ and $\operatorname{dp}(\beta) \geq 2$. Then the definition of $T(s, \beta)$ does not depend on the choice of the $t \in S$ such that $\operatorname{dp}(t \cdot \beta)=\operatorname{dp}(\beta)-1$.

Proof. We argue by induction on $\operatorname{dp}(\beta)$. We take $t, r \in S, t \neq r$, such that $\operatorname{dp}(t \cdot \beta)=\operatorname{dp}(r \cdot \beta)=\operatorname{dp}(\beta)-1$. By Proposition 2.6, we can write $\left\langle\alpha_{t}, \beta\right\rangle=b>0$ and $\left\langle\alpha_{r}, \beta\right\rangle=c>0$.

Case 1: $\left\langle\alpha_{s}, \alpha_{t}\right\rangle=0,\left\langle\alpha_{s}, \alpha_{r}\right\rangle=0$, and $\left\langle\alpha_{t}, \alpha_{r}\right\rangle=0$. We cannot have $\operatorname{dp}(\beta)=2$ in this case. Suppose $\operatorname{dp}(\beta) \geq 3$. Then, by induction,

$$
\begin{aligned}
y \cdot T\left(s, \beta-b \alpha_{t}\right) & =y^{2} \cdot T\left(s, \beta-b \alpha_{t}-c \alpha_{r}\right) & & \text { by (D4) } \\
& =y \cdot T\left(s, \beta-c \alpha_{r}\right) & & \text { by (D4). }
\end{aligned}
$$

Case 2: $\left\langle\alpha_{s}, \alpha_{t}\right\rangle=0,\left\langle\alpha_{s}, \alpha_{r}\right\rangle=0$, and $\left\langle\alpha_{t}, \alpha_{r}\right\rangle=-1$. Suppose $\operatorname{dp}(\beta)=2$. Then $\beta=\alpha_{t}+\alpha_{r}, b=c=1$, and

$$
y \cdot T\left(s, \beta-\alpha_{t}\right)=y \cdot T\left(s, \alpha_{r}\right)=0=y \cdot T\left(s, \alpha_{t}\right)=y \cdot T\left(s, \beta-\alpha_{r}\right) \quad \text { by (D2). }
$$

We cannot have $\operatorname{dp}(\beta)=3$ in this case. Suppose $\operatorname{dp}(\beta) \geq 4$. Then, by induction

$$
\begin{aligned}
y \cdot T\left(s, \beta-b \alpha_{t}\right) & =y^{2} \cdot T\left(s, \beta-b \alpha_{t}-(b+c) \alpha_{r}\right) & & \text { by (D4) } \\
& =y^{3} \cdot T\left(s, \beta-(b+c) \alpha_{t}-(b+c) \alpha_{r}\right) & & \text { by (D4) } \\
& =y \cdot T\left(s, \beta-c \alpha_{r}\right) & & \text { by symmetry. }
\end{aligned}
$$

Case 3: $\left\langle\alpha_{s}, \alpha_{t}\right\rangle=0,\left\langle\alpha_{s}, \alpha_{r}\right\rangle=-1$, and $\left\langle\alpha_{t}, \alpha_{r}\right\rangle=0$. We cannot have $\operatorname{dp}(\beta) \leq 3$ in this case. Suppose $\operatorname{dp}(\beta) \geq 4$. Then, by induction,

$$
\begin{array}{rlrl}
y \cdot T\left(s, \beta-b \alpha_{t}\right)= & y(y-1) \cdot T\left(s, \beta-b \alpha_{t}-c \alpha_{r}\right) & \\
& +y^{2} \cdot T\left(r, \beta-c \alpha_{s}-b \alpha_{t}-c \alpha_{r}\right) & & \text { by (D5) } \\
= & y^{\operatorname{dp}(\beta)-1}(y-1)^{2} & \\
& +y^{2} \cdot T\left(r, \beta-c \alpha_{s}-b \alpha_{t}-c \alpha_{r}\right) & \text { by (D3) } \\
= & (y-1) \cdot T\left(s, \beta-c \alpha_{r}\right)+y \cdot T\left(r, \beta-c \alpha_{s}-c \alpha_{r}\right) & \text { by (D3) and (D4). }
\end{array}
$$

Case 4: $\left\langle\alpha_{s}, \alpha_{t}\right\rangle=0,\left\langle\alpha_{s}, \alpha_{r}\right\rangle=-1$, and $\left\langle\alpha_{t}, \alpha_{r}\right\rangle=-1$. We cannot have $\operatorname{dp}(\beta) \leq 5$ in this case. Suppose $\operatorname{dp}(\beta) \geq 6$. Then, by induction,

$$
\begin{aligned}
& y \cdot T\left(s, \beta-b \alpha_{t}\right) \\
& =y(y-1) \cdot T\left(s, \beta-b \alpha_{t}-(b+c) \alpha_{r}\right) \\
& +y^{2} \cdot T\left(r, \beta-(b+c) \alpha_{s}-b \alpha_{t}-(b+c) \alpha_{r}\right)
\end{aligned}
$$




$$
\begin{aligned}
& =y^{\mathrm{dp}(\beta)-1}(y-1)^{2}+y^{2}(y-1) \text {. } \\
& \cdot T\left(r, \beta-(b+c) \alpha_{s}-(b+c) \alpha_{t}-(b+c) \alpha_{r}\right) \\
& +y^{3} \cdot T\left(t, \beta-(b+c) \alpha_{s}-(b+c) \alpha_{t}-(b+2 c) \alpha_{r}\right) \quad \text { by (D3) and (D5) } \\
& =y^{\mathrm{dp}(\beta)-1}(y-1)^{2}+y^{\mathrm{dp}(\beta)-2}(y-1)^{2} \\
& +y^{3} \cdot T\left(t, \beta-(b+c) \alpha_{s}-(b+c) \alpha_{t}-(b+2 c) \alpha_{r}\right) \quad \text { by (D3) } \\
& =y^{\mathrm{dp}(\beta)-1}(y-1)^{2}+y(y-1) \cdot T\left(r, \beta-c \alpha_{s}-(b+c) \alpha_{t}-c \alpha_{r}\right) \\
& +y^{2} \cdot T\left(t, \beta-c \alpha_{s}-(b+c) \alpha_{t}-(b+2 c) \alpha_{r}\right) \quad \text { by (D3) and (D4) } \\
& =(y-1) \cdot T\left(s, \beta-c \alpha_{r}\right)+y \cdot T\left(r, \beta-c \alpha_{s}-c \alpha_{r}\right) \quad \text { by (D3) and (D5). }
\end{aligned}
$$

Case 5: $\left\langle\alpha_{s}, \alpha_{t}\right\rangle=-1,\left\langle\alpha_{s}, \alpha_{r}\right\rangle=-1$, and $\left\langle\alpha_{t}, \alpha_{r}\right\rangle=0$. We cannot have $\operatorname{dp}(\beta) \leq 5$ in this case. Suppose $\operatorname{dp}(\beta) \geq 6$. Then, by induction,

$$
\begin{array}{rlrl}
(y-1) \cdot T\left(s, \beta-b \alpha_{t}\right)+y \cdot T\left(t, \beta-b \alpha_{s}-b \alpha_{t}\right) & & \\
= & y^{\mathrm{dp}(\beta)-1}(y-1)^{2} & & \\
& +y^{2} \cdot T\left(t, \beta-b \alpha_{s}-b \alpha_{t}-(b+c) \alpha_{r}\right) & & \text { by (D3) and (D4) } \\
= & y^{\mathrm{dp}(\beta)-1}(y-1)^{2}+y^{2}(y-1) & & \\
& \cdot T\left(t, \beta-(b+c) \alpha_{s}-b \alpha_{t}-(b+c) \alpha_{r}\right) & & \\
& +y^{3} \cdot T\left(s, \beta-(b+c) \alpha_{s}-(b+c) \alpha_{t}-(b+c) \alpha_{r}\right) & & \text { by (D5) } \\
= & y^{\mathrm{dp}(\beta)-1}(y-1)^{2}+y^{\mathrm{dp}(\beta)-2}(y-1)^{2} & & \\
& +y^{3} \cdot T\left(s, \beta-(b+c) \alpha_{s}-(b+c) \alpha_{t}-(b+c) \alpha_{r}\right) & & \text { by (D3) } \\
= & (y-1) \cdot T\left(s, \beta-c \alpha_{r}\right)+y \cdot T\left(r, \beta-c \alpha_{s}-c \alpha_{r}\right) & & \text { by symmetry. }
\end{array}
$$

Lemma 3.4. Let $s \in S$ and $\beta \in \Phi^{+}$such that $\operatorname{dp}(\beta) \geq 2$ and $\left\langle\alpha_{s}, \beta\right\rangle=-a<0$. Then the definition of $T(s, \beta)$ does not depend on the choice of the $t \in S$ such that $\operatorname{dp}(t \cdot \beta)=\operatorname{dp}(\beta)-1$.

Proof. We argue by induction on $\operatorname{dp}(\beta)$. We take $t, r \in S, t \neq r$, such that $\operatorname{dp}(t \cdot \beta)=\operatorname{dp}(r \cdot \beta)=\operatorname{dp}(\beta)-1$. By Proposition 2.6, we can write $\left\langle\alpha_{t}, \beta\right\rangle=b>0$ and $\left\langle\alpha_{r}, \beta\right\rangle=c>0$.

Case 1: $\left\langle\alpha_{s}, \alpha_{t}\right\rangle=0,\left\langle\alpha_{s}, \alpha_{r}\right\rangle=0$, and $\left\langle\alpha_{t}, \alpha_{r}\right\rangle=0$. We cannot have $\operatorname{dp}(\beta)=2$ in this case. Suppose $\operatorname{dp}(\beta) \geq 3$. Then, by induction,

$$
\begin{aligned}
y \cdot T\left(s, \beta-b \alpha_{t}\right) & =y^{2} \cdot T\left(s, \beta-b \alpha_{t}-c \alpha_{r}\right) & & \text { by (D6) } \\
& =y \cdot T\left(s, \beta-c \alpha_{r}\right) & & \text { by (D6). }
\end{aligned}
$$

Case 2: $\left\langle\alpha_{s}, \alpha_{t}\right\rangle=0,\left\langle\alpha_{s}, \alpha_{r}\right\rangle=0$, and $\left\langle\alpha_{t}, \alpha_{r}\right\rangle=-1$. We cannot have $\operatorname{dp}(\beta) \leq 3$ in this case. Suppose $\operatorname{dp}(\beta) \geq 4$. Then, by induction, 


$$
\begin{aligned}
y \cdot T\left(s, \beta-b \alpha_{t}\right) & =y^{2} \cdot T\left(s, \beta-b \alpha_{t}-(b+c) \alpha_{r}\right) & & \text { by (D6) } \\
& =y^{3} \cdot T\left(s, \beta-(b+c) \alpha_{t}-(b+c) \alpha_{r}\right) & & \text { by (D6) } \\
& =y \cdot T\left(s, \beta-c \alpha_{r}\right) & & \text { by symmetry. }
\end{aligned}
$$

Case 3: $\left\langle\alpha_{s}, \alpha_{t}\right\rangle=0,\left\langle\alpha_{s}, \alpha_{r}\right\rangle=-1,\left\langle\alpha_{t}, \alpha_{r}\right\rangle=0$, and $c>a$. We cannot have $\operatorname{dp}(\beta) \leq 3$ in this case. Suppose $\operatorname{dp}(\beta) \geq 4$. Then, by induction,

$$
\begin{array}{rlrl}
y & \cdot T\left(s, \beta-b \alpha_{t}\right) & \\
= & y(y-1) \cdot T\left(s, \beta-b \alpha_{t}-c \alpha_{r}\right) & \\
& +y^{2} \cdot T\left(r, \beta-(c-a) \alpha_{s}-b \alpha_{t}-c \alpha_{r}\right) & & \text { by (D7) } \\
= & y^{\mathrm{dp}(\beta)-1}(y-1)^{2} & \\
& +y^{2} \cdot T\left(r, \beta-(c-a) \alpha_{s}-b \alpha_{t}-c \alpha_{r}\right) & & \text { by (D3) } \\
= & (y-1) \cdot T\left(s, \beta-c \alpha_{r}\right)+y \cdot T\left(r, \beta-(c-a) \alpha_{s}-c \alpha_{r}\right) & & \text { by (D3) and (D6). }
\end{array}
$$

Case 4: $\left\langle\alpha_{s}, \alpha_{t}\right\rangle=0,\left\langle\alpha_{s}, \alpha_{r}\right\rangle=-1,\left\langle\alpha_{t}, \alpha_{r}\right\rangle=0$, and $c=a$. We cannot have $\operatorname{dp}(\beta)=2$ in this case. Suppose $\operatorname{dp}(\beta) \geq 3$. Then, by induction,

$$
\begin{array}{ll}
y \cdot T\left(s, \beta-b \alpha_{t}\right) & \\
=y \cdot T\left(r, \beta-b \alpha_{t}-a \alpha_{r}\right)+y(y-1) \cdot T\left(s, \beta-b \alpha_{t}-a \alpha_{r}\right) & \text { by (D8) } \\
=T\left(r, \beta-a \alpha_{r}\right)+(y-1) \cdot T\left(s, \beta-a \alpha_{r}\right) & \text { by (D6) and (D4). }
\end{array}
$$

Case 5: $\left\langle\alpha_{s}, \alpha_{t}\right\rangle=0,\left\langle\alpha_{s}, \alpha_{r}\right\rangle=-1,\left\langle\alpha_{t}, \alpha_{r}\right\rangle=0$, and $c<a$. We cannot have $\operatorname{dp}(\beta)=2$ in this case. Suppose $\operatorname{dp}(\beta) \geq 3$. Then, by induction,

$$
\begin{array}{ll}
y \cdot T\left(s, \beta-b \alpha_{t}\right) & \\
=y^{2} \cdot T\left(s, \beta-b \alpha_{t}-c \alpha_{r}\right)+y \cdot T\left(r, \beta-b \alpha_{t}-c \alpha_{r}\right)+y^{\operatorname{dp}(\beta)-1}(1-y) & \text { by (D9) } \\
=y \cdot T\left(s, \beta-c \alpha_{r}\right)+T\left(r, \beta-c \alpha_{r}\right)+y^{\operatorname{dp}(\beta)-1}(1-y) & \text { by (D6). }
\end{array}
$$

Case 6: $\left\langle\alpha_{s}, \alpha_{t}\right\rangle=0,\left\langle\alpha_{s}, \alpha_{r}\right\rangle=-1,\left\langle\alpha_{t}, \alpha_{r}\right\rangle=-1$, and $c>a$. We cannot have $\operatorname{dp}(\beta) \leq 5$ in this case. Suppose $\operatorname{dp}(\beta) \geq 6$. Then, by induction,

$$
\begin{aligned}
y & \cdot T\left(s, \beta-b \alpha_{t}\right) & \\
= & y(y-1) \cdot T\left(s, \beta-b \alpha_{t}-(b+c) \alpha_{r}\right) & \\
& +y^{2} \cdot T\left(r, \beta-(b+c-a) \alpha_{s}-b \alpha_{t}-(b+c) \alpha_{r}\right) & \\
= & y^{\operatorname{dp}(\beta)-1}(y-1)^{2}+y^{2}(y-1) \cdot & \\
& \cdot T\left(r, \beta-(b+c-a) \alpha_{s}-(b+c) \alpha_{t}-(b+c) \alpha_{r}\right) & \\
& +y^{3} \cdot T\left(t, \beta-(b+c-a) \alpha_{s}-(b+c) \alpha_{t}-(b+2 c-a) \alpha_{r}\right) & \text { by (D3) and (D7) } \\
= & y^{\operatorname{dp}(\beta)-1}(y-1)^{2}+y^{\operatorname{dp}(\beta)-2}(y-1)^{2} & \\
& +y^{3} \cdot T\left(t, \beta-(b+c-a) \alpha_{s}-(b+c) \alpha_{t}-(b+2 c-a) \alpha_{r}\right) & \text { by (D3) }
\end{aligned}
$$




$$
\begin{aligned}
= & y^{\operatorname{dp}(\beta)-1}(y-1)^{2}+y(y-1) . \\
& \cdot T\left(r, \beta-(c-a) \alpha_{s}-(b+c) \alpha_{t}-c \alpha_{r}\right) \\
& +y^{2} \cdot T\left(t, \beta-(c-a) \alpha_{s}-(b+c) \alpha_{t}-(b+2 c-a) \alpha_{r}\right) \quad \text { by (D3) and (D6) } \\
= & (y-1) \cdot T\left(s, \beta-c \alpha_{r}\right)+y \cdot T\left(r, \beta-(c-a) \alpha_{s}-c \alpha_{r}\right) \quad \text { by (D3) and (D7). }
\end{aligned}
$$

Case 7: $\left\langle\alpha_{s}, \alpha_{t}\right\rangle=0,\left\langle\alpha_{s}, \alpha_{r}\right\rangle=-1,\left\langle\alpha_{t}, \alpha_{r}\right\rangle=-1$, and $c=a$. Suppose $\operatorname{dp}(\beta)=2$. Then $a=b=c=1, \beta=\alpha_{t}+\alpha_{r}$, and

$$
\begin{aligned}
y \cdot T\left(s, \beta-\alpha_{t}\right) & =y \cdot T\left(s, \alpha_{r}\right)=0 & & \text { by (D2) } \\
T\left(r, \beta-\alpha_{r}\right)+(y-1) \cdot T\left(s, \beta-\alpha_{r}\right) & =T\left(r, \alpha_{t}\right)+(y-1) \cdot T\left(s, \alpha_{t}\right)=0 & & \text { by (D2). }
\end{aligned}
$$

We cannot have $\operatorname{dp}(\beta) \in\{3,4\}$ in this case. Suppose $\operatorname{dp}(\beta) \geq 5$. Then, by induction,

$$
\begin{array}{rlrl}
y & \cdot T\left(s, \beta-b \alpha_{t}\right) & & \\
= & y(y-1) \cdot T\left(s, \beta-b \alpha_{t}-(b+a) \alpha_{r}\right) & & \text { by (D7) } \\
& +y^{2} \cdot T\left(r, \beta-b \alpha_{s}-b \alpha_{t}-(b+a) \alpha_{r}\right) & & \\
= & y^{\operatorname{dp}(\beta)-1}(y-1)^{2}+y^{2} \cdot T\left(t, \beta-b \alpha_{s}-(b+a) \alpha_{t}-(b+a) \alpha_{r}\right) & & \\
& +y^{2}(y-1) \cdot T\left(r, \beta-b \alpha_{s}-(b+a) \alpha_{t}-(b+a) \alpha_{r}\right) & & \text { by (D3) and (D8) } \\
= & y^{\operatorname{dp}(\beta)-2}(y-1)^{2}+y^{2} \cdot T\left(t, \beta-b \alpha_{s}-(b+a) \alpha_{t}-(b+a) \alpha_{r}\right) & & \\
& +y^{\operatorname{dp}(\beta)-2}(y-1)^{3} & & \\
& +y^{2}(y-1) \cdot T\left(r, \beta-b \alpha_{s}-(b+a) \alpha_{t}-(b+a) \alpha_{r}\right) & \\
= & y^{\operatorname{dp}(\beta)-2}(y-1)^{2}+y^{2} \cdot T\left(t, \beta-b \alpha_{s}-(b+a) \alpha_{t}-(b+a) \alpha_{r}\right) & \\
& +y(y-1)^{2} \cdot T\left(s, \beta-(b+a) \alpha_{t}-(b+a) \alpha_{r}\right) & & \\
& +y^{2}(y-1) \cdot T\left(r, \beta-b \alpha_{s}-(b+a) \alpha_{t}-(b+a) \alpha_{r}\right) & & \\
= & y^{\operatorname{dp}(\beta)-2}(y-1)^{2} & & \\
& +y^{2} \cdot T\left(t, \beta-b \alpha_{s}-(b+a) \alpha_{t}-(b+a) \alpha_{r}\right) & \\
& +y(y-1) \cdot T\left(s, \beta-(b+a) \alpha_{t}-a \alpha_{r}\right) & & \\
= & (y-1) \cdot T\left(r, \beta-(b+a) \alpha_{t}-a \alpha_{r}\right) & & \\
& +y \cdot T\left(t, \beta-(b+a) \alpha_{t}-(b+a) \alpha_{r}\right) & & \\
& +y(y-1) \cdot T\left(s, \beta-(b+a) \alpha_{t}-a \alpha_{r}\right) & & \\
= & T\left(r, \beta-a \alpha_{r}\right)+(y-1) \cdot T\left(s, \beta-a \alpha_{r}\right) & &
\end{array}
$$

Case 8: $\left\langle\alpha_{s}, \alpha_{t}\right\rangle=0,\left\langle\alpha_{s}, \alpha_{r}\right\rangle=-1,\left\langle\alpha_{t}, \alpha_{r}\right\rangle=-1, c<a$, and $b+c>a$. We cannot have $\operatorname{dp}(\beta) \leq 4$ in this case. Suppose $\operatorname{dp}(\beta) \geq 5$. Then, by induction, 


$$
\begin{aligned}
& y \cdot T\left(s, \beta-b \alpha_{t}\right) \\
& =y(y-1) \cdot T\left(s, \beta-b \alpha_{t}-(b+c) \alpha_{r}\right) \\
& +y^{2} \cdot T\left(r, \beta-(b+c-a) \alpha_{s}-b \alpha_{t}-(b+c) \alpha_{r}\right) \quad \text { by (D7) } \\
& =y^{\mathrm{dp}(\beta)-1}(y-1)^{2}+y^{3} \text {. } \\
& \cdot T\left(r, \beta-(b+c-a) \alpha_{s}-(b+c) \alpha_{t}-(b+c) \alpha_{r}\right) \\
& +y^{2} \cdot T\left(t, \beta-(b+c-a) \alpha_{s}-(b+c) \alpha_{t}-(b+c) \alpha_{r}\right) \\
& +y^{\mathrm{dp}(\beta)-2}(1-y) \\
& =y^{\mathrm{dp}(\beta)-1}(y-1)^{2} \\
& +y^{3} \cdot T\left(r, \beta-(b+c-a) \alpha_{s}-(b+c) \alpha_{t}-(b+c) \alpha_{r}\right) \\
& +y^{\mathrm{dp}(\beta)-2}(y-1)^{2} \\
& +y^{2} \cdot T\left(t, \beta-(b+c-a) \alpha_{s}-(b+c) \alpha_{t}-(b+c) \alpha_{r}\right) \\
& +y^{\mathrm{dp}(\beta)-1}(1-y) \\
& =y^{2}(y-1) \cdot T\left(s, \beta-(b+c) \alpha_{t}-(b+c) \alpha_{r}\right) \\
& +y^{3} \cdot T\left(r, \beta-(b+c-a) \alpha_{s}-(b+c) \alpha_{t}-(b+c) \alpha_{r}\right) \\
& +y^{\mathrm{dp}(\beta)-2}(y-1)^{2} \\
& +y \cdot T\left(t, \beta-(b+c) \alpha_{t}-(b+c) \alpha_{r}\right)+y^{\mathrm{dp}(\beta)-1}(1-y) \quad \text { by (D3) and (D6) } \\
& =y^{2} \cdot T\left(s, \beta-(b+c) \alpha_{t}-c \alpha_{r}\right)+(y-1) \text {. } \\
& \cdot T\left(r, \beta-(b+c) \alpha_{t}-c \alpha_{r}\right) \\
& +y \cdot T\left(t, \beta-(b+c) \alpha_{t}-(b+c) \alpha_{r}\right)+y^{\mathrm{dp}(\beta)-1}(1-y) \quad \text { by (D7) and (D3) } \\
& =y \cdot T\left(s, \beta-c \alpha_{r}\right)+T\left(r, \beta-c \alpha_{r}\right)+y^{\mathrm{dp}(\beta)-1}(1-y) \quad \text { by (D6) and (D7). }
\end{aligned}
$$

Case 9: $\left\langle\alpha_{s}, \alpha_{t}\right\rangle=0,\left\langle\alpha_{s}, \alpha_{r}\right\rangle=-1,\left\langle\alpha_{t}, \alpha_{r}\right\rangle=-1, c<a$, and $b+c=a$. We cannot have $\operatorname{dp}(\beta) \leq 3$ in this case. Suppose $\operatorname{dp}(\beta) \geq 4$. Then, by induction,

$$
\begin{array}{rlr}
y & T\left(s, \beta-b \alpha_{t}\right) & \\
= & y \cdot T\left(r, \beta-b \alpha_{t}-a \alpha_{r}\right)+y(y-1) \cdot T\left(s, \beta-b \alpha_{t}-a \alpha_{r}\right) & \text { by (D8) } \\
= & y^{2} \cdot T\left(r, \beta-a \alpha_{t}-a \alpha_{r}\right)+y \cdot T\left(t, \beta-a \alpha_{t}-a \alpha_{r}\right) & \\
& +y^{\operatorname{dp}(\beta)-2}(1-y) & \\
& +y^{2}(y-1) \cdot T\left(s, \beta-a \alpha_{t}-a \alpha_{r}\right) & \\
= & y^{2} \cdot T\left(r, \beta-a \alpha_{t}-a \alpha_{r}\right)+y^{2}(y-1) \cdot & \\
& \cdot T\left(s, \beta-a \alpha_{t}-a \alpha_{r}\right)+y^{\operatorname{dp}(\beta)-2}(y-1)^{2} & \\
& +y \cdot T\left(t, \beta-a \alpha_{t}-a \alpha_{r}\right)+y^{\operatorname{dp}(\beta)-1}(1-y) &
\end{array}
$$




$$
\begin{aligned}
= & y^{2} \cdot T\left(s, \beta-a \alpha_{t}-c \alpha_{r}\right)+(y-1) \cdot T\left(r, \beta-a \alpha_{t}-c \alpha_{r}\right) & & \\
& +y \cdot T\left(t, \beta-a \alpha_{t}-a \alpha_{r}\right)+y^{\operatorname{dp}(\beta)-1}(1-y) & & \text { by (D8) and (D3) } \\
= & y \cdot T\left(s, \beta-c \alpha_{r}\right)+T\left(r, \beta-c \alpha_{r}\right)+y^{\operatorname{dp}(\beta)-1}(1-y) & & \text { by (D6) and (D7). }
\end{aligned}
$$

Case 10: $\left\langle\alpha_{s}, \alpha_{t}\right\rangle=0,\left\langle\alpha_{s}, \alpha_{r}\right\rangle=-1,\left\langle\alpha_{t}, \alpha_{r}\right\rangle=-1, c<a$, and $b+c<a$. We cannot have $\operatorname{dp}(\beta) \leq 3$ in this case. Suppose $\operatorname{dp}(\beta) \geq 4$. Then, by induction,

$$
\begin{array}{rlr}
y & \cdot T\left(s, \beta-b \alpha_{t}\right) & \\
= & y^{2} \cdot T\left(s, \beta-b \alpha_{t}-(b+c) \alpha_{r}\right) & \\
& +y \cdot T\left(r, \beta-b \alpha_{t}-(b+c) \alpha_{r}\right) & \\
& +y^{\mathrm{dp}(\beta)-1}(1-y) & \\
= & y^{3} \cdot T\left(s, \beta-(b+c) \alpha_{t}-(b+c) \alpha_{r}\right) & \\
& +y^{2} \cdot T\left(r, \beta-(b+c) \alpha_{t}-(b+c) \alpha_{r}\right) & \\
& +y \cdot T\left(t, \beta-(b+c) \alpha_{t}-(b+c) \alpha_{r}\right) & \\
& +y^{\mathrm{dp}(\beta)-2}(1-y)+y^{\mathrm{dp}(\beta)-1}(1-y) & \\
= & y^{3} \cdot T\left(s, \beta-(b+c) \alpha_{t}-(b+c) \alpha_{r}\right) & \\
& +y^{2} \cdot T\left(r, \beta-(b+c) \alpha_{t}-(b+c) \alpha_{r}\right) & \\
& +y^{\mathrm{dp}(\beta)-1}(1-y)+y^{\mathrm{dp}(\beta)-2}(y-1)^{2} & \\
& +y \cdot T\left(t, \beta-(b+c) \alpha_{t}-(b+c) \alpha_{r}\right)+y^{\mathrm{dp}(\beta)-1}(1-y) & \\
= & y^{2} \cdot T\left(s, \beta-(b+c) \alpha_{t}-c \alpha_{r}\right) & \\
& +(y-1) \cdot T\left(r, \beta-(b+c) \alpha_{t}-c \alpha_{r}\right) & \\
& +y \cdot T\left(t, \beta-(b+c) \alpha_{t}-(b+c) \alpha_{r}\right)+y^{\mathrm{dp}(\beta)-1}(1-y) & \\
= & y \cdot T\left(s, \beta-c \alpha_{r}\right)+T\left(r, \beta-c \alpha_{r}\right)+y^{\operatorname{dp}(\beta)-1}(1-y) & \text { by (D6) and (D7). }
\end{array}
$$

Case 11: $\left\langle\alpha_{s}, \alpha_{t}\right\rangle=-1,\left\langle\alpha_{s}, \alpha_{r}\right\rangle=-1,\left\langle\alpha_{t}, \alpha_{r}\right\rangle=0, b>a$, and $c>a$. We cannot have $\operatorname{dp}(\beta) \leq 5$ in this case. Suppose $\operatorname{dp}(\beta) \geq 6$. Then, by induction,

$$
\begin{array}{rlrl}
(y-1) \cdot T\left(s, \beta-b \alpha_{t}\right)+y \cdot T\left(t, \beta-(b-a) \alpha_{s}-b \alpha_{t}\right) & & \\
= & y^{\mathrm{dp}(\beta)-1}(y-1)^{2}+y^{2} . & \\
& \cdot T\left(t, \beta-(b-a) \alpha_{s}-b \alpha_{t}-(b+c-a) \alpha_{r}\right) & & \text { by (D3) and (D6) } \\
= & y^{\mathrm{dp}(\beta)-1}(y-1)^{2}+y^{2}(y-1) \cdot & \\
& \cdot T\left(t, \beta-(b+c-a) \alpha_{s}-b \alpha_{t}-(b+c-a) \alpha_{r}\right) & \\
& +y^{3} \cdot T\left(s, \beta-(b+c-a) \alpha_{s}-(b+c-a) \alpha_{t}-(b+c-a) \alpha_{r}\right) & & \text { by (D7) } \\
= & y^{\operatorname{dp}(\beta)-1}(y-1)^{2}+y^{\operatorname{dp}(\beta)-2}(y-1)^{2}+y^{3} . & \\
& \cdot T\left(s, \beta-(b+c-a) \alpha_{s}-(b+c-a) \alpha_{t}-(b+c-a) \alpha_{r}\right) & & \text { by (D3) }
\end{array}
$$


$=(y-1) \cdot T\left(s, \beta-c \alpha_{r}\right)+y \cdot T\left(r, \beta-(c-a) \alpha_{s}-c \alpha_{r}\right) \quad$ by symmetry.

Case 12: $\left\langle\alpha_{s}, \alpha_{t}\right\rangle=-1,\left\langle\alpha_{s}, \alpha_{r}\right\rangle=-1,\left\langle\alpha_{t}, \alpha_{r}\right\rangle=0$, and $b>c=a$. We cannot have $\operatorname{dp}(\beta) \leq 4$ in this case. Suppose $\operatorname{dp}(\beta) \geq 5$. Then, by induction,

$$
\begin{array}{rlrl}
(y-1) \cdot T\left(s, \beta-b \alpha_{t}\right)+y \cdot T\left(t, \beta-(b-a) \alpha_{s}-b \alpha_{t}\right) & & \\
= & y^{\mathrm{dp}(\beta)-1}(y-1)^{2}+y^{2} \cdot T\left(t, \beta-(b-a) \alpha_{s}-b \alpha_{t}-b \alpha_{r}\right) & & \text { by (D3) and (D6) } \\
= & y^{\mathrm{dp}(\beta)-1}(y-1)^{2}+y^{2} \cdot & & \\
& \cdot T\left(s, \beta-b \alpha_{s}-b \alpha_{t}-b \alpha_{r}\right) & & \text { by (D8) } \\
& +y^{2}(y-1) \cdot T\left(t, \beta-b \alpha_{s}-b \alpha_{t}-b \alpha_{r}\right) & & \\
= & y^{\mathrm{dp}(\beta)-2}(y-1)^{2}+y^{2} \cdot T\left(s, \beta-b \alpha_{s}-b \alpha_{t}-b \alpha_{r}\right) & & \\
& +y^{\mathrm{dp}(\beta)-2}(y-1)^{3} & & \\
& +y^{2}(y-1) \cdot T\left(t, \beta-b \alpha_{s}-b \alpha_{t}-b \alpha_{r}\right) & \\
= & y(y-1) \cdot T\left(r, \beta-b \alpha_{s}-b \alpha_{t}-a \alpha_{r}\right) & & \\
& +y^{2} \cdot T\left(s, \beta-b \alpha_{s}-b \alpha_{t}-b \alpha_{r}\right)+y^{\mathrm{dp}(\beta)-2}(y-1)^{3} & & \\
& +y(y-1) \cdot T\left(t, \beta-b \alpha_{s}-b \alpha_{t}-a \alpha_{r}\right) & & \\
= & y \cdot T\left(r, \beta-b \alpha_{t}-a \alpha_{r}\right)+(y-1)^{2} \cdot T\left(s, \beta-b \alpha_{t}-a \alpha_{r}\right) & & \\
& +y(y-1) \cdot T\left(t, \beta-b \alpha_{s}-b \alpha_{t}-a \alpha_{r}\right) & & \text { by (D7) and (D3) } \\
= & T\left(r, \beta-a \alpha_{r}\right)+(y-1) \cdot T\left(s, \beta-a \alpha_{r}\right) & & \text { by (D6) and (D5). }
\end{array}
$$

Case 13: $\left\langle\alpha_{s}, \alpha_{t}\right\rangle=-1,\left\langle\alpha_{s}, \alpha_{r}\right\rangle=-1,\left\langle\alpha_{t}, \alpha_{r}\right\rangle=0$, and $b>a>c$. We cannot have $\operatorname{dp}(\beta) \leq 3$ in this case. Suppose $\operatorname{dp}(\beta) \geq 4$. Then, by induction,

$$
\begin{aligned}
(y & -1) \cdot T\left(s, \beta-b \alpha_{t}\right)+y \cdot T\left(t, \beta-(b-a) \alpha_{s}-b \alpha_{t}\right) \\
= & y^{\mathrm{dp}(\beta)-1}(y-1)^{2} \\
& +y^{2} \cdot T\left(t, \beta-(b-a) \alpha_{s}-b \alpha_{t}-(b+c-a) \alpha_{r}\right) \\
= & y^{\mathrm{dp}(\beta)-1}(y-1)^{2} \\
& +y^{3} \cdot T\left(t, \beta-(b+c-a) \alpha_{s}-b \alpha_{t}-(b+c-a) \alpha_{r}\right) \\
& +y^{2} \cdot T\left(s, \beta-(b+c-a) \alpha_{s}-b \alpha_{t}-(b+c-a) \alpha_{r}\right) \\
& +y^{\mathrm{dp}(\beta)-2}(1-y) \\
= & y^{\mathrm{dp}(\beta)-1}(y-1)^{2} \\
& +y^{3} \cdot T\left(t, \beta-(b+c-a) \alpha_{s}-b \alpha_{t}-(b+c-a) \alpha_{r}\right) \\
& +y^{\mathrm{dp}(\beta)-2}(y-1)^{2} \\
& +y^{2} \cdot T\left(s, \beta-(b+c-a) \alpha_{s}-b \alpha_{t}-(b+c-a) \alpha_{r}\right) \\
& +y^{\operatorname{dp}(\beta)-1}(1-y)
\end{aligned}
$$




$$
\begin{aligned}
= & y^{\mathrm{dp}(\beta)-1}(y-1)^{2}+y^{2} \cdot T\left(t, \beta-(b+c-a) \alpha_{s}-b \alpha_{t}-c \alpha_{r}\right) \\
& +y(y-1) \cdot T\left(r, \beta-(b+c-a) \alpha_{s}-b \alpha_{t}-c \alpha_{r}\right) \\
& +y^{2} \cdot T\left(s, \beta-(b+c-a) \alpha_{s}-b \alpha_{t}-(b+c-a) \alpha_{r}\right) \\
& +y^{\mathrm{dp}(\beta)-1}(1-y) \\
= & y(y-1) \cdot T\left(s, \beta-b \alpha_{t}-c \alpha_{r}\right) \\
& +y^{2} \cdot T\left(t, \beta-(b+c-a) \alpha_{s}-b \alpha_{t}-c \alpha_{r}\right) \\
& +y \cdot T\left(r, \beta-b \alpha_{t}-c \alpha_{r}\right)+y^{\operatorname{dp}(\beta)-1}(1-y) \\
= & y \cdot T\left(s, \beta-c \alpha_{r}\right)+T\left(r, \beta-c \alpha_{r}\right)+y^{\mathrm{dp}(\beta)-1}(1-y)
\end{aligned}
$$

Case 14: $\left\langle\alpha_{s}, \alpha_{t}\right\rangle=-1,\left\langle\alpha_{s}, \alpha_{r}\right\rangle=-1,\left\langle\alpha_{t}, \alpha_{r}\right\rangle=0$, and $a=b=c$. We cannot have $\operatorname{dp}(\beta) \leq 3$ in this case. Suppose $\operatorname{dp}(\beta) \geq 4$. Then, by induction,

$$
\begin{array}{rlrl}
T( & \left.t, \beta-a \alpha_{t}\right)+(y-1) \cdot T\left(s, \beta-a \alpha_{t}\right) & & \\
= & y \cdot T\left(t, \beta-a \alpha_{t}-a \alpha_{r}\right)+(y-1)^{2} \cdot T\left(s, \beta-a \alpha_{t}-a \alpha_{r}\right) & & \\
& +y(y-1) \cdot T\left(r, \beta-a \alpha_{s}-a \alpha_{t}-a \alpha_{r}\right) & & \text { by (D6) and (D5) } \\
= & y \cdot T\left(s, \beta-a \alpha_{s}-a \alpha_{t}-a \alpha_{r}\right) & & \\
& +y(y-1) \cdot T\left(t, \beta-a \alpha_{s}-a \alpha_{t}-a \alpha_{r}\right) & \\
& +y^{\operatorname{dp}(\beta)-2}(y-1)^{3}+y(y-1) \cdot T\left(r, \beta-a \alpha_{s}-a \alpha_{t}-a \alpha_{r}\right) & & \text { by (D8) and (D3) } \\
= & T\left(r, \beta-a \alpha_{r}\right)+(y-1) \cdot T\left(s, \beta-a \alpha_{r}\right) & & \text { by symmetry. }
\end{array}
$$

Case 15: $\left\langle\alpha_{s}, \alpha_{t}\right\rangle=-1,\left\langle\alpha_{s}, \alpha_{r}\right\rangle=-1,\left\langle\alpha_{t}, \alpha_{r}\right\rangle=0$, and $a=b>c$. We cannot have $\operatorname{dp}(\beta) \leq 3$ in this case. Suppose $\operatorname{dp}(\beta) \geq 4$. Then, by induction,

$$
\begin{array}{rlr}
T( & \left.t, \beta-a \alpha_{t}\right)+(y-1) \cdot T\left(s, \beta-a \alpha_{t}\right) & \\
= & y \cdot T\left(t, \beta-a \alpha_{t}-c \alpha_{r}\right)+(y-1)^{2} \cdot T\left(s, \beta-a \alpha_{t}-c \alpha_{r}\right) & \\
& +y(y-1) \cdot T\left(r, \beta-c \alpha_{s}-a \alpha_{t}-c \alpha_{r}\right) & \\
= & y^{2} \cdot T\left(t, \beta-c \alpha_{s}-a \alpha_{t}-c \alpha_{r}\right) & \\
& +y \cdot T\left(s, \beta-c \alpha_{s}-a \alpha_{t}-c \alpha_{r}\right)+y^{\mathrm{dp}(\beta)-2}(1-y) & \\
& +y^{\mathrm{dp}(\beta)-2}(y-1)^{3}+y(y-1) \cdot T\left(r, \beta-c \alpha_{s}-a \alpha_{t}-c \alpha_{r}\right) & \text { by (D9) and (D) } \\
= & y^{\mathrm{dp}(\beta)-1}(y-1)^{2}+y^{2} \cdot T\left(t, \beta-c \alpha_{s}-a \alpha_{t}-c \alpha_{r}\right) & \\
& +y \cdot T\left(s, \beta-c \alpha_{s}-a \alpha_{t}-c \alpha_{r}\right) & \\
& +y(y-1) \cdot T\left(r, \beta-c \alpha_{s}-a \alpha_{t}-c \alpha_{r}\right)+y^{\mathrm{dp}(\beta)-1}(1-y) & \\
= & y(y-1) \cdot T\left(s, \beta-a \alpha_{t}-c \alpha_{r}\right) & \\
& +y^{2} \cdot T\left(t, \beta-c \alpha_{s}-a \alpha_{t}-c \alpha_{r}\right) & \\
& +y \cdot T\left(r, \beta-a \alpha_{t}-c \alpha_{r}\right)+y^{\operatorname{dp}(\beta)-1}(1-y) & \text { by (D3) and (D8) }
\end{array}
$$




$$
=y \cdot T\left(s, \beta-c \alpha_{r}\right)+T\left(r, \beta-c \alpha_{r}\right)+y^{\mathrm{dp}(\beta)-1}(1-y) \quad \text { by (D7) and (D6). }
$$

Case 16: $\left\langle\alpha_{s}, \alpha_{t}\right\rangle=-1,\left\langle\alpha_{s}, \alpha_{r}\right\rangle=-1,\left\langle\alpha_{t}, \alpha_{r}\right\rangle=0, a>b, a>c$, and $b+c>a$. We cannot have $\operatorname{dp}(\beta) \leq 3$ in this case. Suppose $\operatorname{dp}(\beta) \geq 4$. Then, by induction,

$$
\begin{array}{rlrl}
y & \cdot T\left(s, \beta-b \alpha_{t}\right)+T\left(t, \beta-b \alpha_{t}\right)+y^{\mathrm{dp}(\beta)-1}(1-y) & \\
= & y(y-1) \cdot T\left(s, \beta-b \alpha_{t}-c \alpha_{r}\right) & & \\
& +y^{2} \cdot T\left(r, \beta-(b+c-a) \alpha_{s}-b \alpha_{t}-c \alpha_{r}\right) & & \\
& +y \cdot T\left(t, \beta-b \alpha_{t}-c \alpha_{r}\right)+y^{\operatorname{dp}(\beta)-1}(1-y) & & \text { by (D7) and (D6) } \\
= & y^{\mathrm{dp}(\beta)-1}(y-1)^{2}+y^{2} \cdot T\left(r, \beta-(b+c-a) \alpha_{s}-b \alpha_{t}-c \alpha_{r}\right) & \\
& +y^{2} \cdot T\left(t, \beta-(b+c-a) \alpha_{s}-b \alpha_{t}-c \alpha_{r}\right) & \\
& +y \cdot T\left(s, \beta-(b+c-a) \alpha_{s}-b \alpha_{t}-c \alpha_{r}\right)+y^{\mathrm{dp}(\beta)-2}(1-y) & \\
& +y^{\operatorname{dp}(\beta)-1}(1-y) & & \text { by (D3) and (D9) } \\
= & y \cdot T\left(s, \beta-c \alpha_{r}\right)+T\left(r, \beta-c \alpha_{r}\right)+y^{\operatorname{dp}(\beta)-1}(1-y) & & \text { by symmetry. }
\end{array}
$$

Case 17: $\left\langle\alpha_{s}, \alpha_{t}\right\rangle=-1,\left\langle\alpha_{s}, \alpha_{r}\right\rangle=-1,\left\langle\alpha_{t}, \alpha_{r}\right\rangle=0$, and $a=b+c$. We cannot have $\operatorname{dp}(\beta)=2$ in this case. Suppose $\operatorname{dp}(\beta) \geq 3$. Then, by induction,

$$
\begin{aligned}
& y \cdot T\left(s, \beta-b \alpha_{t}\right)+T\left(t, \beta-b \alpha_{t}\right)+y^{\mathrm{dp}(\beta)-1}(1-y) \\
& =y \cdot T\left(r, \beta-b \alpha_{t}-c \alpha_{r}\right)+y(y-1) \cdot T\left(s, \beta-b \alpha_{t}-c \alpha_{r}\right) \\
& +y \cdot T\left(t, \beta-b \alpha_{t}-c \alpha_{r}\right)+y^{\mathrm{dp}(\beta)-1}(1-y) \quad \text { by (D8) and (D6) } \\
& =y \cdot T\left(s, \beta-c \alpha_{r}\right)+T\left(r, \beta-c \alpha_{r}\right)+y^{\mathrm{dp}(\beta)-1}(1-y) \quad \text { by symmetry. }
\end{aligned}
$$

Case 18: $\left\langle\alpha_{s}, \alpha_{t}\right\rangle=-1,\left\langle\alpha_{s}, \alpha_{r}\right\rangle=-1,\left\langle\alpha_{t}, \alpha_{r}\right\rangle=0$, and $a>b+c$. We cannot have $\operatorname{dp}(\beta)=2$ in this case. Suppose $\operatorname{dp}(\beta) \geq 3$. Then, by induction,

$$
\begin{aligned}
& y \cdot T\left(s, \beta-b \alpha_{t}\right)+T\left(t, \beta-b \alpha_{t}\right)+y^{\mathrm{dp}(\beta)-1}(1-y) \\
& =y^{2} \cdot T\left(s, \beta-b \alpha_{t}-c \alpha_{r}\right) \\
& +y \cdot T\left(r, \beta-b \alpha_{t}-c \alpha_{r}\right)+y^{\mathrm{dp}(\beta)-1}(1-y) \\
& +y \cdot T\left(t, \beta-b \alpha_{t}-c \alpha_{r}\right)+y^{\mathrm{dp}(\beta)-1}(1-y) \quad \text { by (D9) and (D6) } \\
& =y \cdot T\left(s, \beta-c \alpha_{r}\right)+T\left(r, \beta-c \alpha_{r}\right)+y^{\operatorname{dp}(\beta)-1}(1-y) \quad \text { by symmetry. }
\end{aligned}
$$

\section{References}

[Alt] J. Altobelli, The word problem for Artin groups of FC type, J. Pure Appl. Algebra 129 (1998), 1-22. 
[Big] S. Bigelow, Braid groups are linear, J. Amer. Math. Soc. 14 (2001), 471-486.

[BS] E. Brieskorn and K. Saito, Artin-Gruppen und Coxeter-Gruppen, Invent. Math. 17 (1972), 245-271.

$[\mathrm{BH}] \quad$ B. Brink and R. B. Howlett, A finiteness property and an automatic structure for Coxeter groups, Math. Ann. 296 (1993), 179-190.

[Cha] R. Charney, Injectivity of the positive monoid for some infinite type Artin groups, Cossey, John (ed.) et al., Geometric group theory down under, Proceedings of a special year in geometric group theory, Canberra, Australia, July 14-19, 1996. De Gruyter, Berlin, 1999, 103-118.

[ChP] J. R. Cho and S. J. Pride, Embedding semigroups into groups, and the asphericity of semigroups, Int. J. Algebra Comput. 3 (1993), 1-13.

[CW] A. M. Cohen and D. B. Wales, Linearity of Artin groups of finite type, Israel J. Math., to appear.

[Cri] J. Crisp, Injective maps between Artin groups, Cossey, John (ed.) et al., Geometric group theory down under, Proceedings of a special year in geometric group theory, Canberra, Australia, July 14-19, 1996. De Gruyter, Berlin, 1999, 119-137.

[CrP] J. Crisp and L. Paris, The solution to a conjecture of Tits on the subgroup generated by the squares of the generators of an Artin group, Invent. Math. 145 (2001), 19-36.

[Del] P. Deligne, Les immeubles des groupes de tresses généralisés, Invent. Math. 17 (1972), 273-302.

[Deo] V. V. Deodhar, On the root system of a Coxeter group, Commun. Algebra 10 (1982), 611-630.

[Dig] F. Digne, On the linearity of Artin braid groups, J. Algebra, to appear.

[Hil] H. Hiller, Geometry of Coxeter groups, Research Notes in Mathematics, 54. Pitman Advanced Publishing program, Boston-London-Melbourn, 1982.

[Kra1] D. Krammer, The braid group $B_{4}$ is linear, Invent. Math. 142 (2000), 451-486.

[Kra2] D. Krammer, Braid groups are linear, Ann. Math. 155 (2002), 131-156.

[Mic] J. Michel, A note on words in braid monoids, J. Algebra 215 (1999), 366-377.

[Tit1] J. Tits, Le problème des mots dans les groupes de Coxeter, Sympos. Math., Roma 1, Teoria Gruppi, Dic. 1967 e Teoria Continui Polari, Aprile 1968, Academic Press, London, 1969, 175-185.

[Tit2] J. Tits, Normalisateurs de tores. I: Groupes de Coxeter étendus, J. Algebra 4 (1966), $96-116$.

Luis Paris

Université de Bourgogne

Laboratoire de Topologie

UMR $5584 \mathrm{du}$ CNRS

BP 47870

F-21078 Dijon cedex

France

e-mail: lparis@u-bourgogne.fr

(Received: March 14, 2002) 